\title{
L'aménagement du parcours allemand du Rhin Mesures exécutées, en cours et en projet
}

\author{
River training on the German Rhine \\ Past, present and future work \\ PAR M. CASPER \\ PHAESLEN'T DER WASSER- UND SCHIFAHRTSDHEKTON, MANZ
}

\begin{abstract}
La, nue d'ensemble du parcours du Rhin entre Constance ef la frontiore germano-néerlandaise, et en particulier celui en aval de Lauterbura (le parcours en amont de ce point etant trates en détail dans d'autres articles de la présenter publication) met en évidence les travanx d'aménagement réalises dans le passé, surtoul aux $X I X^{\circ}$ ef $X X^{\circ}$ siecles, ainsi que les methodes utilisées et les divers succès enregistrés. I'antenr. montre qu'il importait tout d'abord d'éliminer les effets devasteurs du fleuve, que ces travaux ont également fourni de meilleures conditions pour la navigation, et comment, par la stite, les aménagements intéressant la navigation ont peu à peu occupé le premier plan. Ia 'régularisation du Rhin est pratiquement achevée dans son ensemble, apportant une grande sécurité anx conditions de vie des rinerains et favorisant l'énolution de l'industrie le long du fleuve, ainsi qu'un essol" considérable du trafic.

Quelques amenagements resteront encore à faire en differents endroits, dans l'avenir, alyant pour objet d'une part d'accroitre l'efficacité du Rhin en tant que voie de communication, et d'autre part - et surtout sur le Rhin-Inférieur - de. Intter contre l'érosion. Les mesures encore à prendre sont exposées en détail.
\end{abstract}

En voyant aujourd'hui l'importance énorme de la navigation sur le Rhin et, sur les deux rives tant du Rhin-Supérieur que du Rhin-Inférieur, les grandes villes aux industries très développées, il est difficile de se faire une idée de ce qu'était l'aspect du fleuve et du paysage qu'il traverse, à la fin du $\mathrm{XIX}^{\circ}$ siècle. Des villes connues aujourd'hui dans le monde entier, comme Ludwigshafen, sur le Rhin-Supérieur, ou

\begin{abstract}
In a general survey of the Rhine between Conslance and the Dutch/German border, in which special consideration is given to the section below Lanterburg $\left(^{\star}\right)$ the Anthor discusses past work, especially during the 19 th and 20th cenluries, as well as the river-training methods used and the extent to which they were successful. He shows hom the river first had to be made harmless, which incidentally also produced better navigational conditions, and how navigational requirements gradually became the chief consideration in the various training schemes that were put into effect. The bulk of this work has now been completed, providing the inhabilants of the riverside areas with safe living conditions and giving rise to a considerable industrial development along the Rhine, as well as a tremendous expansion in river traffic.

A few local works will still be required here and there in the future, in order to increase the river's traffic-carrying capacity even further and to prevent erosion, especially on the Lower Rhine. These are discussed in detail.
\end{abstract}

Ruhrort, sur le Rhin-Inférieur - pour n'en citer que deux - n'existaient même pas à cette époque, ou bien elles étaient tout au plus de petits lieux habités très insignifiants. Ces villes doivent leur existence et leur essor non seulement au développement considérable de lindus-

(*) The upstream section is discussed in detail elsewhere in this number. 


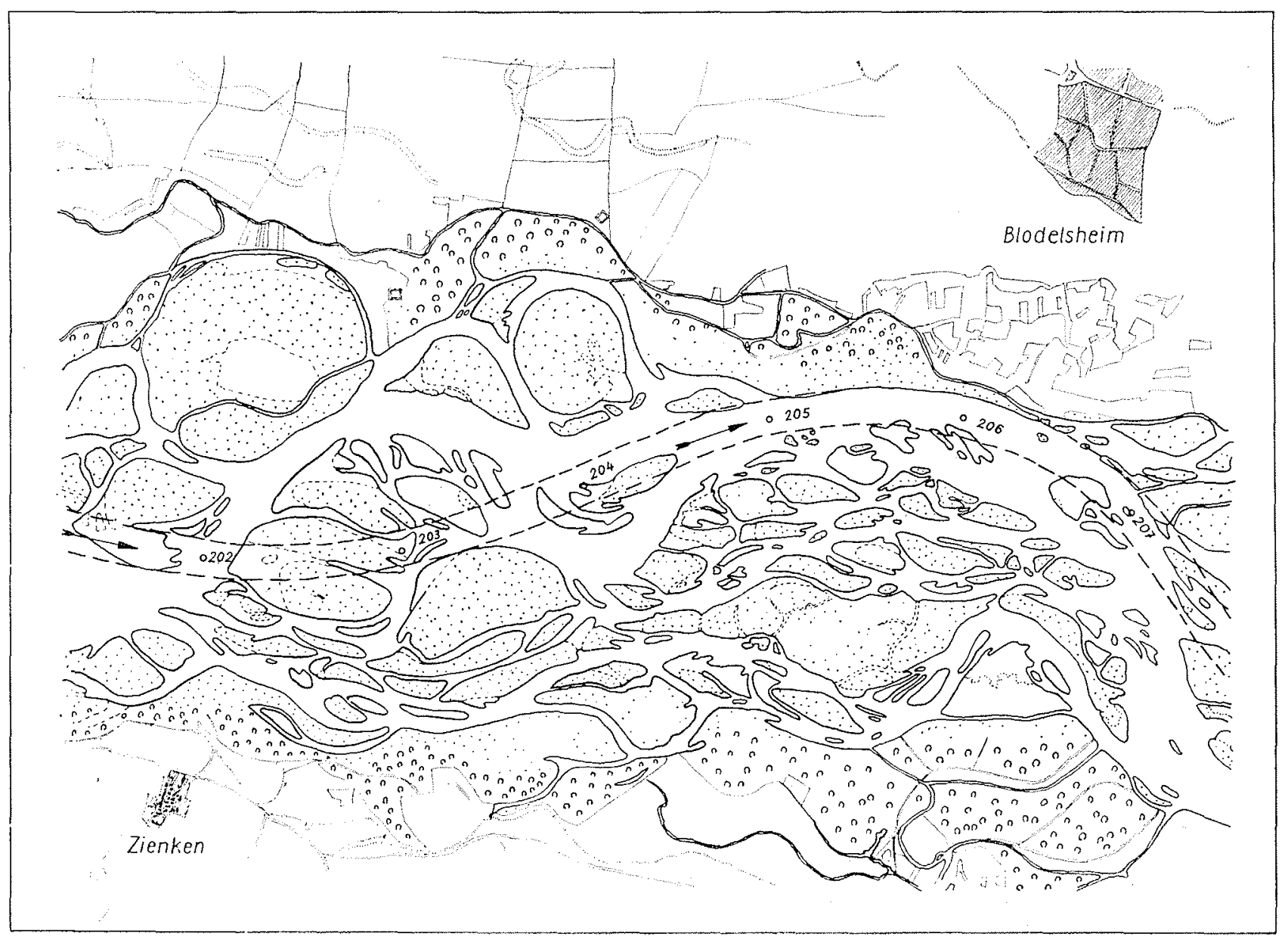

Fu. 1. Ramitications du Rhin superieur avant la regularisation

trie aux $\mathrm{xIx}^{\circ}$ et $\mathrm{xx}^{\prime \prime}$ siècles, mais surtout à leur situation au bord du fleuve qui fut, justement à cette époque, aménagé pour devenir la première artère de communication de l'Europe. Ce qui nous frappe le plus aujourd'hui est l'importance primordiale du Rhin en tant que voie de communication; toutefois il ne faut pas perdre de vue la tâche originale et naturelle de tout fleuve, qui est d'évacuer dans la mer les eaux. de précipitation. Les fleuves représentent un maillon indispensable de celte chaîne qu'est le circuit éternel de l'eau dans la nature, chaîne bienfaisante pour les terres et les habitants riverains, mais aussi parfois dangereusement destructive. L'homme intervenant dans les conditions naturelles de cet écoulement ne restera pas impuni s'il cherche seulement à exploiter ces eaux à certaines fins et non à d'autres; il doit par conséquent tenter d'aboutir à un état de choses permettant encore au fleuve de remplir toutes ses différentes tâches en toute harmonie.

Les efforts des hommes pour se protéger contre la puissance dévastatrice du fleuve furent à l'origine de toutes les mesures d'aménagement. Ces aménagements ont souvent automatiquement entraîné d'eux-mêmes des améliorations favorisant la navigation. Ce ne fut que plus tard, alors que les exigences du trafic fluvial se faisaient sentir de plus en plus, que la navigation passa au premier plan dans toutes les mesures prises pour aménager le fleuve; il s'ensuivit que, maîtrisé dans la mesure des possibilités humaines, comme il l'est aujourd'hui, le Rhin satisfait actuellement de plus en plus aux exigences du trafic moderne, grâce aux aménagements toujours plus étendus que l'on y réalise.

Les habitants de la vallée du Rhin ont cherché depuis des siècles à maîtriser la puissance des eaux et à améliorer la navigabilité du fleuve en construisant des ouvrages d'art le long de son cours. Or, les conditions politiques et économiques de ces époques d'antan étaient telles que les mesures prises restaient obligatoirement d'une importance purement locale et ne constituaient que des solutions de courte durée. On ne peut parler d'un aménagement systématique du 
Rhin - exception faite d'un certain nombre de mesures réalisées sur le Rhin-Inférieur - qu'à partir de l'an 1800, car ce ne fut qu'après cette date que les conditions politiques se stabilisèrent suffisamment pour permettre à des organisations régionales efficaces de prendre en main les aménagements nécessaires.

Le Hatul-Rhin, entre le lac de Constance et Bâle, s'écoule la plupart du temps entre de hautes berges escarpées, dans un lit bien endigué; grâce aux capacités de retenue des lacs de Constance et de l'Aar, son débit est relativement uniforme, de sorte que l'on n'a commencé a entreprendre des travaux d'aménagement importants qu'à l'époque où l'on se mit à créer des retenues pour la production d'énergie. Ces aménagements sont présentés dans l'article de M. Kolly.

En aval de Bâle, jusqu'à la région de Mayence, le Rhin passe par le fossé tectonicue constituant la basse-plaine de la Haute-Rhénanie, qui comporte des galets et des graviers allant jusqu'à des profondeurs très importantes. Le soussol est très mobile, de sorte qu'en période de crue, le fleuve rompait souvent ses rives sans pro- tection, dévastant les terres en les arrachant ou en les exhaussant. Surtout dans la partie sud, dans la région Bade-Alsace, le fleuve s'était ramifié en un grand nombre de petits chenaux (voir fig. 1) qui changeaient perpétuellement leurs cours, de sorte qu'il ne pouvait être question d'une navigation régulière. Ailleurs, dans la parlie nord, c'est-à-dire dans la région Bade-Palatinal, où le fleuve était contenu dans un seul chenal plus ou moins défini, il traçait de vastes méandres d'un fond de vallée à l'autre, souvent rompant des berges en période de crue, de sorte que les conditions d'existence des riverains élaient toujours précaires, (voir fig.2). Ce fut le colonel badois Tulla, qui devint par la suite le directeur général des aménagements, qui reconnut le premier la nécessité de baser toute solution viable sur un aménagement conçu selon un plan unifié. A la suite de longues et pénibles négociations entre les deux Etats riverains, c'est-à-dire la France et l'Etat de Bade, pour te parcours Bâle-Lauterburğ, et entre l'Etat de Bade et la Bavière pour le parcours LauterburgMannheim, on parvint enfin à fixer les bases permettant de donner au lleuve un lit bien défini

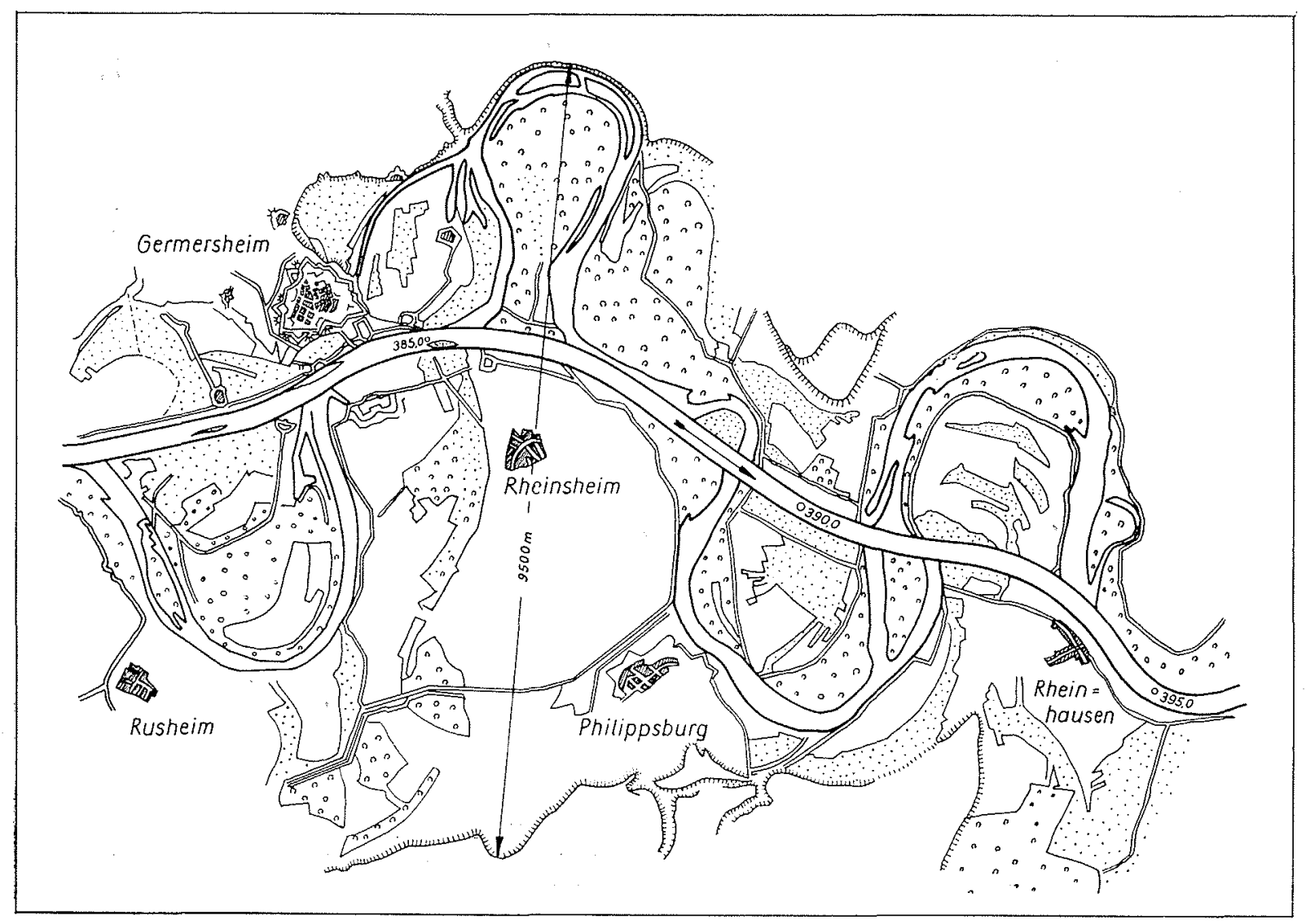

Fig. 2. - Formation de méandres sur le parcours badois/palatinois du fleuve 
aux berges stables. On se heurla également à des difficultés avec les Etats situés plus en aval, c'est-à-dire la Hesse et la Prusse, et mème les Pays-Bas, qui craignaient de voir s'accroître le risque d'inondation, et une augmentation des débits solides sur leurs territoires.

En dépit de toute cette opposition, on atlaqua les lravaux de correction et on les continua jusqu'au milieu du $x x^{*}$ siècle. Nous n'examinerons pas dans cet article le détail des travaux effectués en amont de Lauterburg puisqu'ils ont déjà été exposés ailleurs dans le présent numéro.

Conformément aux accords signés entre l'Etat de Bade et la Bavière, on réunit le fleuve dans un lit unique sur son parcours frontalier entre ces deux Etats, et on élimina les boucles successives en effectuant les coupures convenables. Sur la base des études préliminaires très poussées faites par Tulla, on choisit pour le nouveau lit $\mathrm{du}$ fleuve - qui devait être large de $200 \mathrm{~m}$ à Bâle, el de $250 \mathrm{~m}$ à Strasbourg — la largeur de $240 \mathrm{~m}$ d'une berge à l'autre, ce pour des pentes de talus de $1 / 1,5$ en-dessous de la ligne des moyennes eaux, de $1 / 2$ au-dessus de cette ligne. (La largeurexcédentaire à Strasbourg fut sans doute choisio compte tenu de l'érosion à laquelle on s'attendait sur ce parcours). On ne donna pas tout de suite à ces coupures de boucles leurs largeurs définitives, se contentant de creuser des tranchées et laissant au fleuve lui-même le soin de les élargir par la suite. En aménageant progressivement les ouvrages de fermeture des anciens chenaux d'une manière correspondante, on put diriger le débit de telle façon que l'on arriva à l'état définitif au bout de plusieurs années. Dès que l'on eût arasé les berges presque jusqu'au niveau du plan d'eau normal, on les renforça avec des fascines submergées et remplies de pierres, avec au-dessus un ouvrage longitudinal constitué par des enrochements arrimés et par un perré de berge, le tout allant jusqu'au-dessus du niveau des moyennes eaux. Les parties des talus au-dessus de cette ligne furent alors recou-

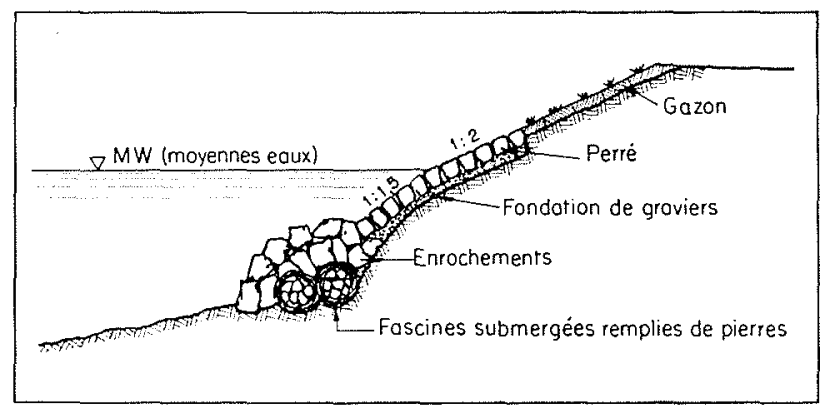

Fici. 3

Aménagement des berges dans les coupures réalisés sue le parcours corrigé vertes de gazon (fig. 3). Les tracés normaux des berges des parcours hors des coupures furent presques tous fixés par des ouvrages parallèles à la rive et reliés à elle par des épis et des ouvrages transversaux (fig. 4). Sur le parcours badois et palatinois, les coupures totalisent uno longueur de $34 \mathrm{~km}$, e'est-à-dire environ les $40 \%$ de la longueur totale aménagée du fleuve, ce qui correspond à un raccourcissement d'environ $53 \mathrm{~km}$ de la distance à parcourir par les bateaux, soit $38 \%$ de la longueur de l'ancien trajet. Les coupures individuelles et leurs années de réalisation sont résumées dans le tableau cidessous :

\begin{tabular}{|c|c|c|}
\hline & 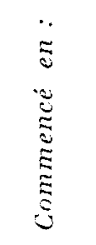 & 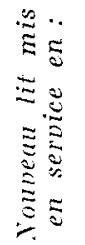 \\
\hline 1. Coupure de Neuburg . . . . . . . & 1817 & 1821 \\
\hline 2. Coupure de Daxland $\left({ }^{*}\right) \ldots \ldots$ & 1819 & 1822 \\
\hline 3. Coupure de Pforz . . . . . . . & 1817 & 1824 \\
\hline 4. Coupure de Knieling ......... & 1817 & 1818 \\
\hline 5. Coupure de Worth .......... & 1818 & 1821 \\
\hline 6. Coupure de Neupfotz. . . . . . . & 1817 & 1828 \\
\hline 7. Coupure de Linkenheim . . . . & 1826 & 1830 \\
\hline 8. Coupure de Leimersheim . . . . . & 1827 & 1837 \\
\hline 9. Coupure de Germersheim. . . . . . & 1826 & 1833 \\
\hline 10. Coupure de Rheinsheim I . . . & 1826 & 1833 \\
\hline 11. Coupure de Rheinsheim $11 \ldots$ & 1826 & 1832 \\
\hline 12. Coupure de Mechtersheim . . . & 1837 & 1844 \\
\hline 13. Coupure d'Oberhausen & & \\
\hline 14. Coupure d'Angelhofen $\ldots \ldots \ldots$ & $\begin{array}{l}1842 \\
1826\end{array}$ & $\begin{array}{l}1844 \\
1879\end{array}$ \\
\hline 15. Coupure d'Otterstalt . . . . . . & 1833 & 1845 \\
\hline 16. Coupure de Ketsch . . . . . . & 1833 & 1839 \\
\hline 17. Coupure d'Altrip ...... & 1865 & 1874 \\
\hline 18. Coupure de Friesenheim ... & 1827 & 1862 \\
\hline $\begin{array}{l}\text { s coupures en italique ont été } \\
\text { ninistrations du Palatinat, et } \\
\text { e Bade. }\end{array}$ & áalisé & $\begin{array}{c}\text { par } \\
\text { par }\end{array}$ \\
\hline
\end{tabular}

Grâce à ces travaux de correction, on a réussi à atteindre entièrement le but désiré, c'est-à-dire d'une part de protéger les terres contre les crues dévastatrices, et d'autre part de maintenir le fleuve dans un lit bien délimité. Notons toutefois que ces mesures ont donné lieu à des érosions considérables et désavantageuses pour les cultures, surtout entre Istein et Brisach.

Le lit de correction réalisé de cette manière était conçu pour l'évacuation en lit fermé du débit de crue estival, ce qui rendait par conséquent ce lit trop large pour les moyennes et basses eaux. Le résultat en fut que, sauf pendant 


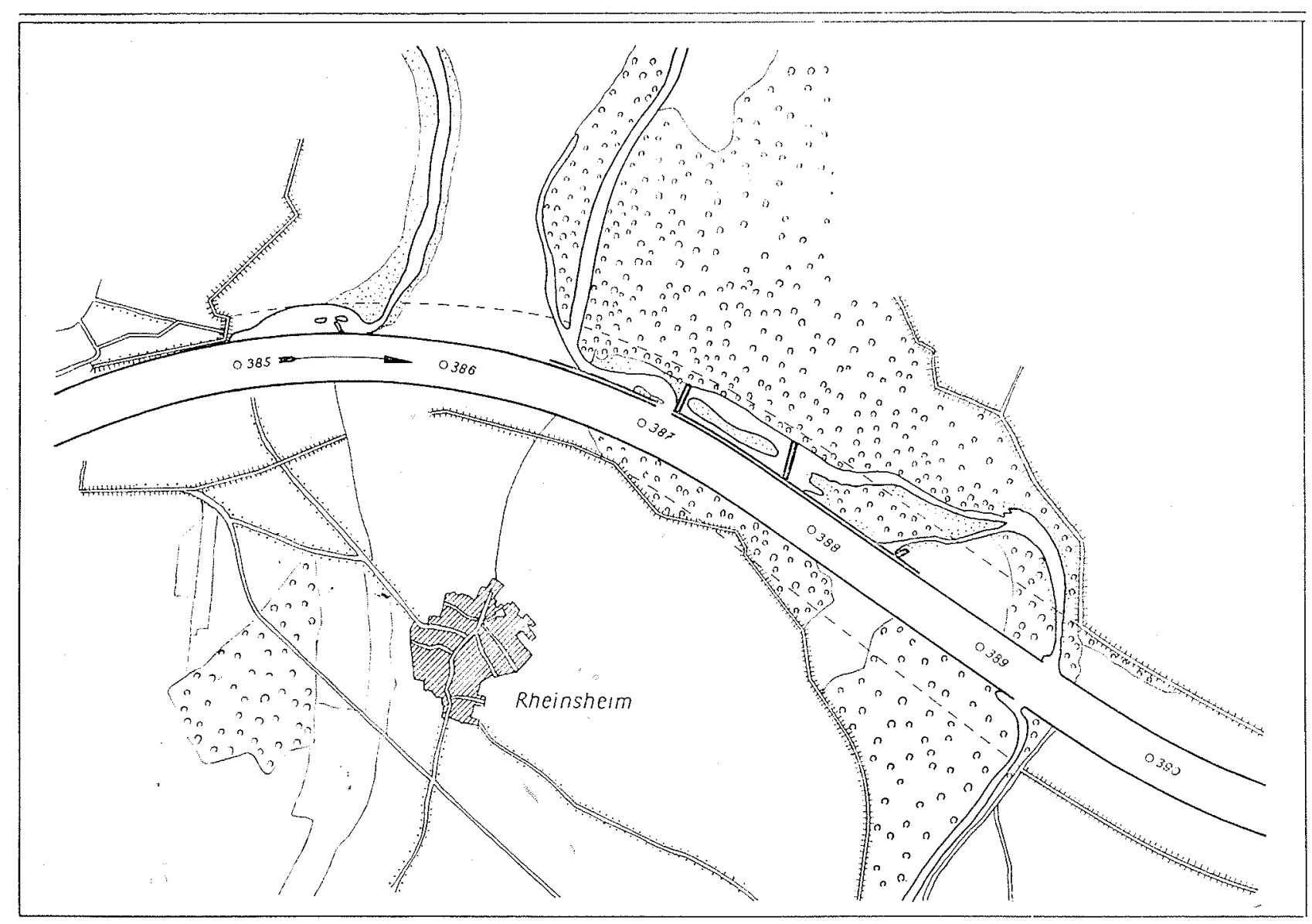

\section{FII. 4}

Stabilisation du tracé des berges sur le parcours non compris dans les coupures, par des ouvrages parallèles

et transversaux.

FIG. 5)

Banes de graviev 《itinerants》 sur le

Rhin-Supéricur

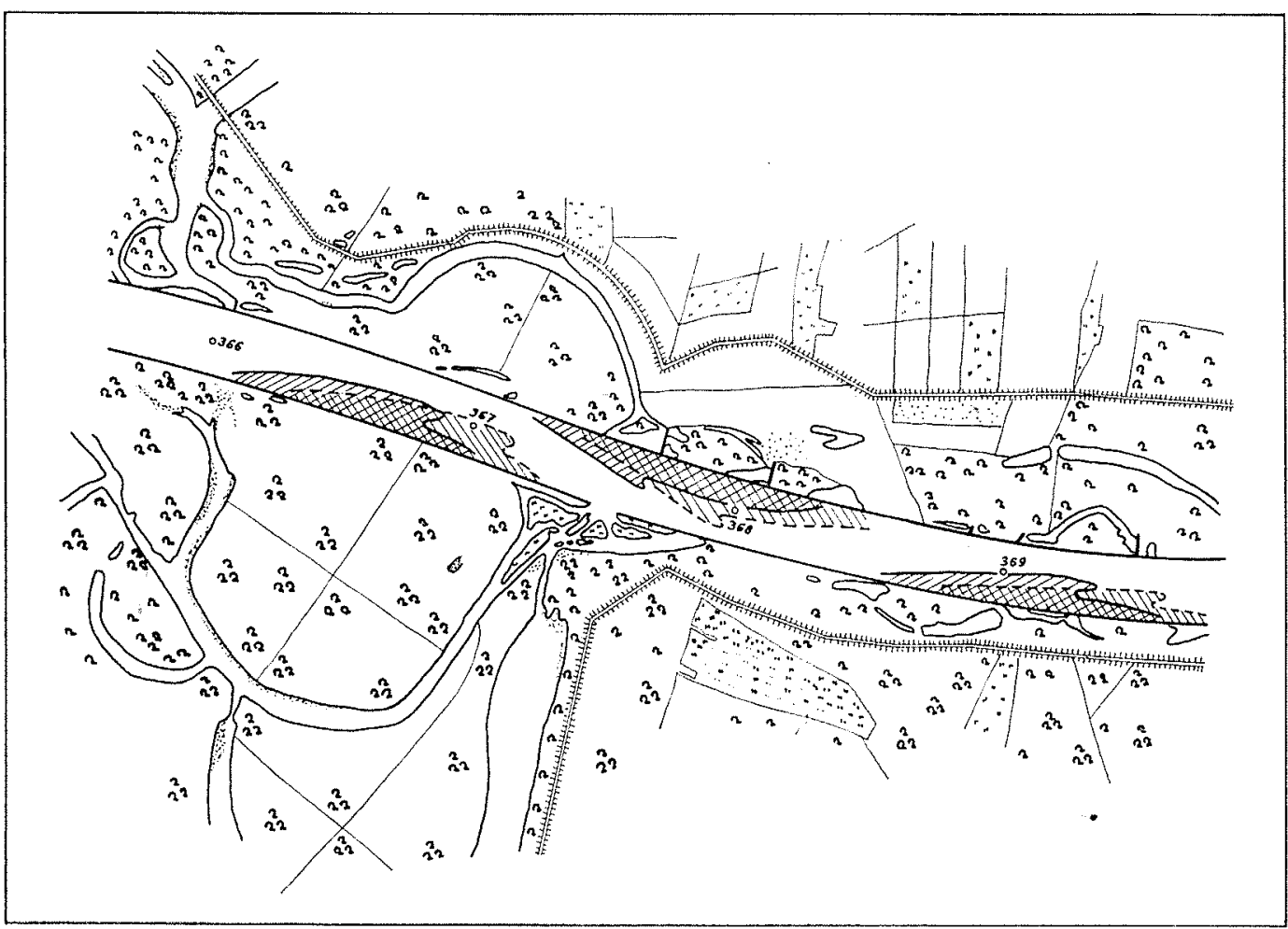




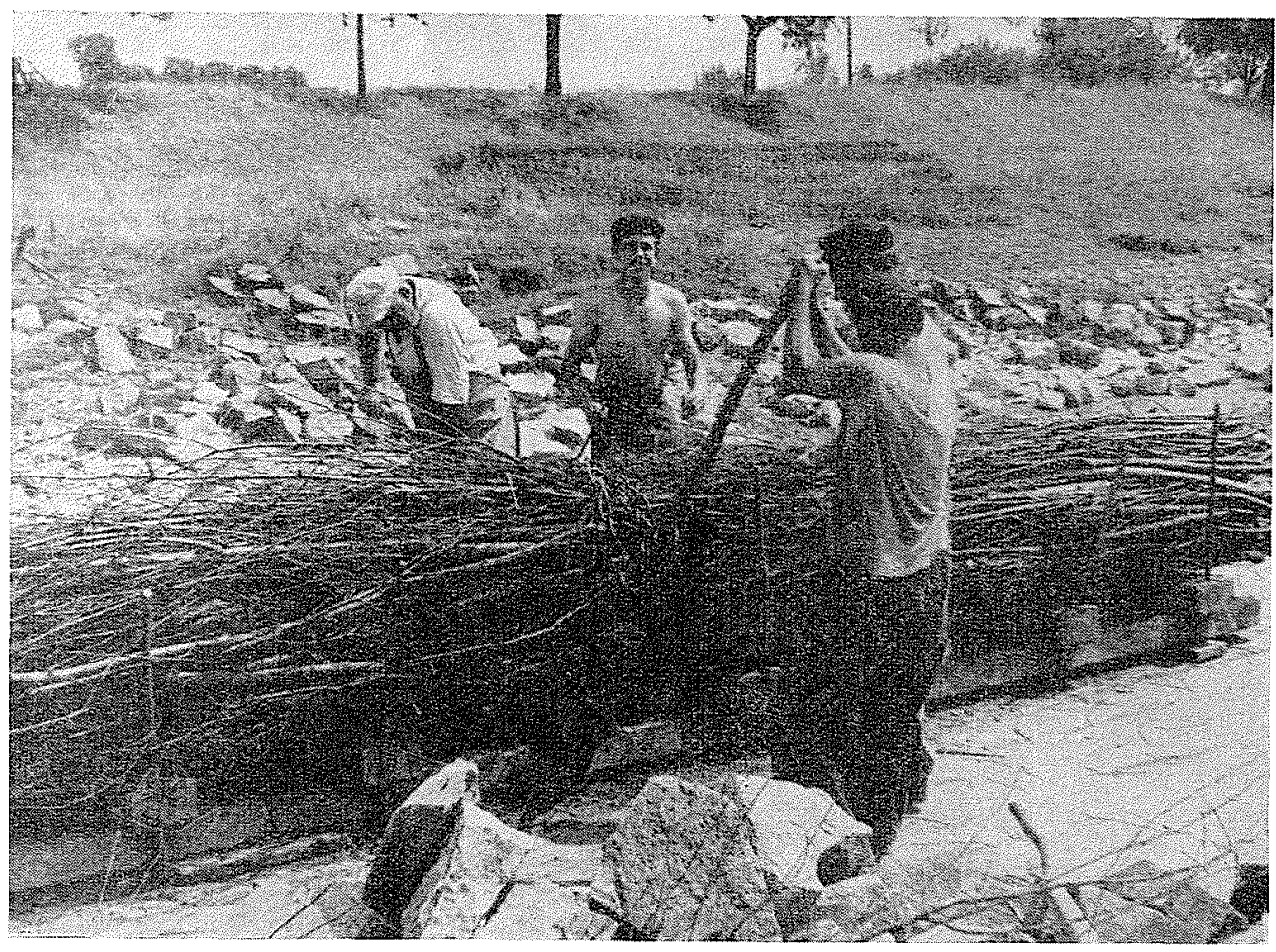

FIG. 6

Serrage

d'une « saucisse constituée d'un:

fascine

ayant un «nyau» de grosses pierres

la crue d'été, le fleuve changeait constamment de cours dans ce lit, creusant des trous profonds et formant des banes de graviers d'une très grande hauteur. De plus, ces banes changeaient tout le temps de position, gênant ainsi considérablement la navigation, sinon même la rendant impossible pendant de longues périodes de l'année. On peut se faire une idée précise de ces bancs itinérants en examinant la figure 5 .

Peu après l'achèvement de ces coupures, l'augmentation du trafic, et par suite, l'emploi de bateaux toujours plus grands, rendirent évidente la nécessité d'améliorer l'état du lit du fleuve de telle façon que les bateaux naviguant jusqu'à Mannheim puissent également être mis en service sur le Rhin-Supérieur jusqu’à Strasbourg, du moins pendant la majeure partie de l'année. On aborda déjà l'étude des deux possibilités ci-après pendant la période suivant l'année 1870: soit aménager le Rhin lui-même encore davantage si possible, soit chercher à résoudre le problème au moyen d'un canal latéral. Les études effectuées par Honsell, directeur des Aménagements de l'Etat de Bade amenèrent à la conviction qu'il était possible d'établir un chenal navigable de profondeur et de largeur suffisantes en effectuant dans le lit du fleuve des travaux de régularisation convenables. Honsell présenta cette possibilité dans un mémoire qu'il publia en 1890, mais il fallut encore attendre lan 1907 avant que les travaux nécessaires ne soient mis en chantier, ceci sur le parcours entre Sondernheim et Strasbourg.

On implanta des épis d'atterrissement et des seuils de fond dans le lit de correction, constituant de la sorte un chenal de navigation tracant des méandres bien ordonnés entre les berges, et assurant le transport continu des ma tériaux solides sans provoquer la formation de banes de dépôts. La largeur de régularisation augmente vers l'aval en fonction du débil. d'abord fixée à $160 \mathrm{~m}$ à la hauteur de Strasbourg, et passant à $180 \mathrm{~m}$ à Sondernheim, ces cotes étant mesurées entre les têtes des épis. Les dimensions correspondant à la cote $-2 \mathrm{~m}$ par rapport à la cote des basses eaux, sont de $120 \mathrm{~m}$ pour Strasbourg et $140 \mathrm{~m}$ pour Sondernheim. D'après l'étude, la profondeur au point le moins profond devait être de $2,0 \mathrm{~m}$, ceci pour la cote de $+3,0 \mathrm{~m}$ mesurée au limnimètre de Maxau. On dispose les épis d'atterrissement de telle sorte que deux épis opposés se situent toujours dans un profil transversal orienté à environ $90^{\circ}$ par rapport à l'axe d'écoulement du fleuve. Sui vant leur position, l'écartement de ces épis entre eux peut varier du simple au triple de leur longueur. Ils sont constitués par des «saucisses? réalisées en constituant des fascines autour d'un «noyau» en grosses pierres. Leur longueur est d'environ $8,0 \mathrm{~m}$, le diamètre de la «saucisse * étant de 0,80 m (fig. 6).

Lors des récents travaux d'entretien, on a en- 
registré de bons succès en remplaçant les fascines formant «l'enveloppe» de ces «saucisses » par des treillis en fil de fer galvanisé. La construction d'un des épis est indiquée sur la fig. 7. Sur les parcours où le lit d'étiage, et par suite le chenal navigable, sont contigus à l'ouvrage de la berge de correction, on a prévu des guideaux orientés dans le sens parallèle à la berge et ayant une pente de $1 / 3$, afin de protéger la structure de la berge et de combler les creux aftouillés dans le lit. De même que les épis, ces guideaux ont également été réalisés en «saucisses». Les travaux de correction sur le parcours Sondernheim-Strasbourg ont été effectués entre les années 1907 et 1915 et ont généralement fourni les bons résultats escomptés.

Peu après l'achèvement de ces travaux, il devint évident que les conditions de navigation sur le parcours entre Sondernheim et Mannheim étaient moins favorables que sur le parcours régularisé en amont de Sondernheim. La première guerre mondiale et l'instabilité des années suivantes ont empêché la reprise des travaux d'aménagement, mais dès le retour de temps plus normaux, on reprit l'étude des avant-projets porlant sur l'aménagemenl de ce deuxième parcours. En se basant sur les expériences obtenues sur le parcours Sondernheim-Strasbourg, on commença par fermer des brèches béantes par des travaux de fondation effectués au niveau des moyennes eaux, (c'est-à-dire des ouvrages parallèles), et là où les terres riveraines s'étaient atterries, on établit des berges normales jusqu'au-dessus du niveau des crues d'été. (Le profil en travers est montré sur la fig. 8). Au moyen d'épis et de seuils de fond, on aménagea ensuite les parcours aux profondeurs les plus défavorables entre Sondernheim et Spire, créant par endroits un lit d'étiage analogue à celui du parcours aménagé plus en amont. Le mode de construction des épis fut semblable à celui utilisé dans le parcours situé en amont.

Ces travaux furent réalisés pendant les années 1926-29 et complétés sur le parcours entre Germersheim et Mechtersheim en 1936 et 1937.

Toutefois, ces mesures ne constituaient toujours pas la régularisation définitive du parcours Sondernheim-Mannheim, car plusieurs troncons intermédiaires, bien que courts, restaient encore à aménager.

Sur le parcours contigu au précédent, de Mannheim à Mayence, les conditions au début du $\mathrm{xIx}^{\circ}$ siècle étaient un peu différentes de celles existant sur les parcours que nous venons d'examiner. Sauf quelques exceptions, les courbes du fleuve ne présentaient plus le même caractère de méandres serrés et étroits que plus loin en amont. Seules deux coupures importantes furent nécessaires, celle au «Geier», pour éliminer la boucle d'Erfelden, et celle à Lampertheim,

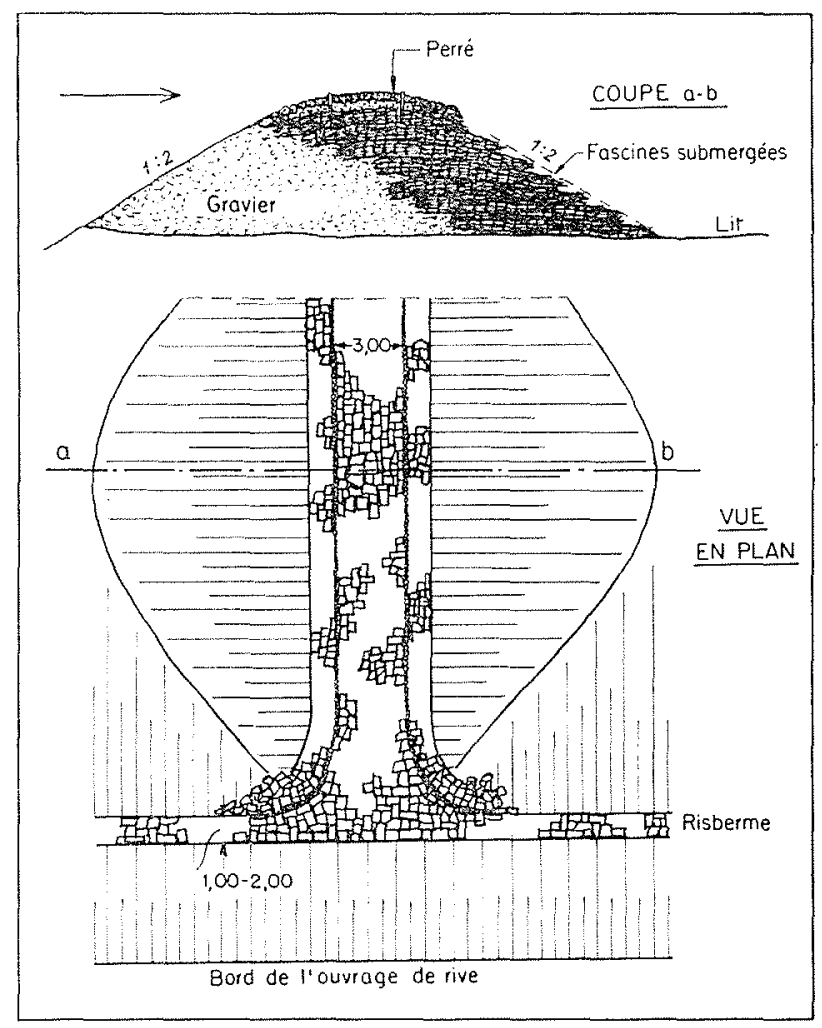

FIG. 7

Régularisation des basses eaux entre Sirrasbourg et Sondernheim. Construction d'un épi

où le fleuve avait déjà rompu ses berges pendant l'hiver 1801/02. Conformément aux plans de Gröhnke, directeur général des Constructions de l'Etat de Hesse, on coupa en 1828/29 la boucle d'Erfelden au moyen de la percée au «Geier», d'une manière analogue aux coupures réalisées plus loin en amont. Déjà pendant l'hiver $1829 / 30$, il fut possible aux bateaux de faibles dimensions d'emprunter cette nouvelle voie. Tou-

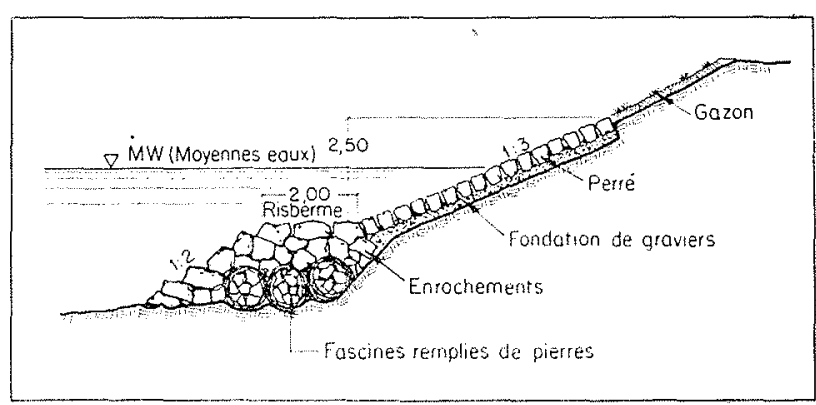

FIG. 8

Rive aménagée normalement pour la régularisation des basses eaux 


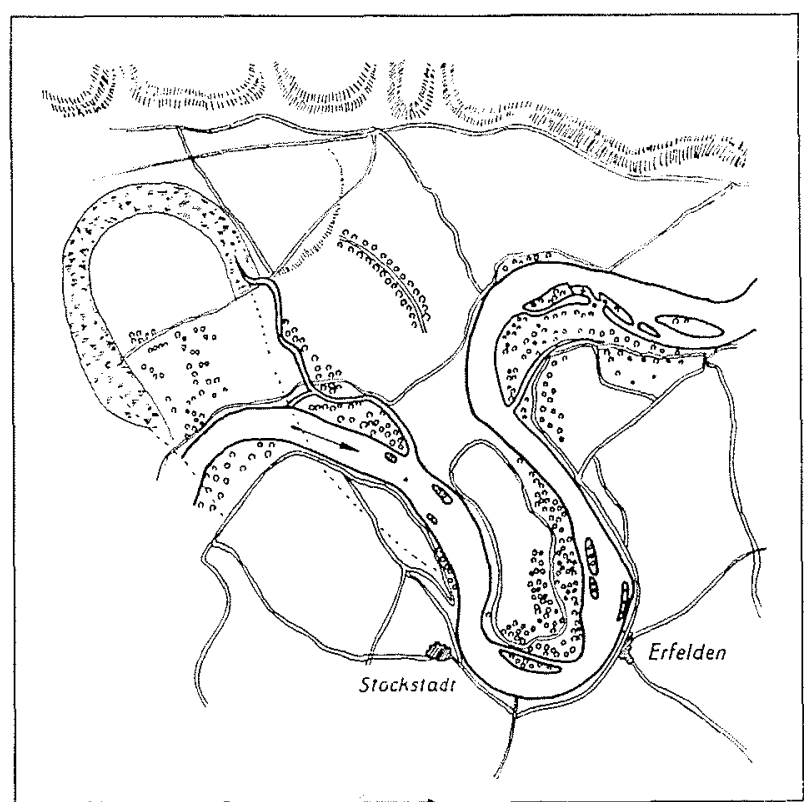

F1G. ?

Coupure au Geyer. - Avant les travaux

tefois, l'élargissement à $300 \mathrm{~m}$ que l'on désirait réaliser sur cette coupure exigea des travaux d'assez longue durée, travaux qui ne se terminèrent finalement qu'en 1836 . Le chemin à parcourir par les bateaux fut ainsi raccourci de $10,5 \mathrm{~km}$. Cette coupure illustrée dans les figures

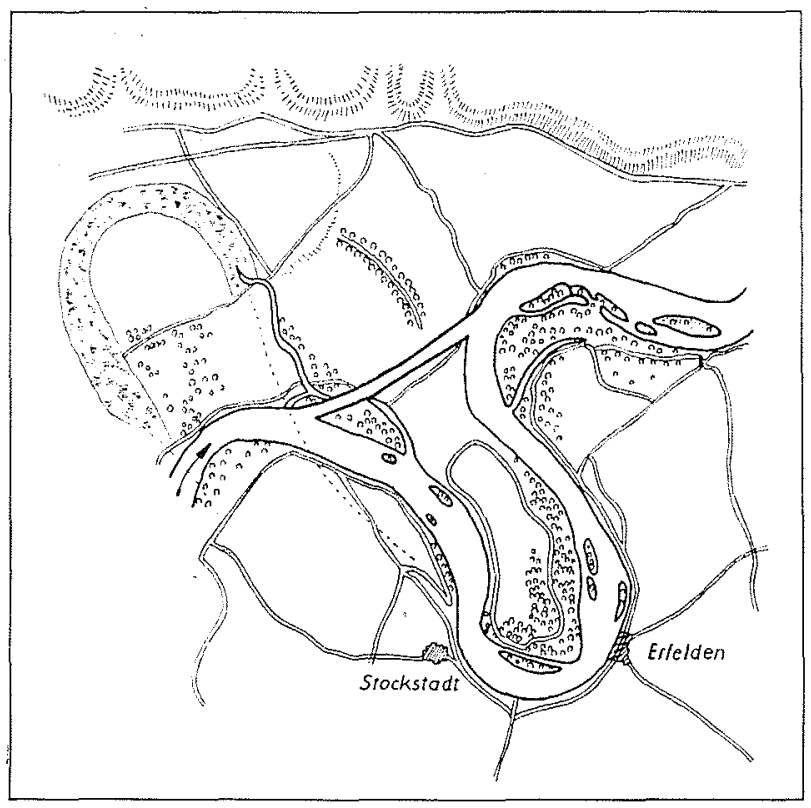

Fr. 10

Coupure au Geyer. - Etat en 1829, pendant les travaux
9 à 11, qui montrent également les terres nouvelles gagnées grâce à l'atterrissement à l'intérieur de l'ancienne boucle. Outre le raccourcissement de la voie de navigation et la meilleure sécurité obtenue, cet aménagement favorisa également nettement les conditions de culture des terres. Par contre, à Lampertheim, plusieurs dizaines d'années devaient s'écouler avant que le projet de coupure dont nous avons parlé plus haut ne se réalise conformément aux plans et que l'on n'aboutisse à un chenal de navigation vraiment convenable. Les travaux de correction commencés en 1879 ne purent être considérés comme terminés que 10 ans plus tard. Outre ces deux coupures, on a effectué des travaux d'aménagement des berges et implanté des ouvrages longi-

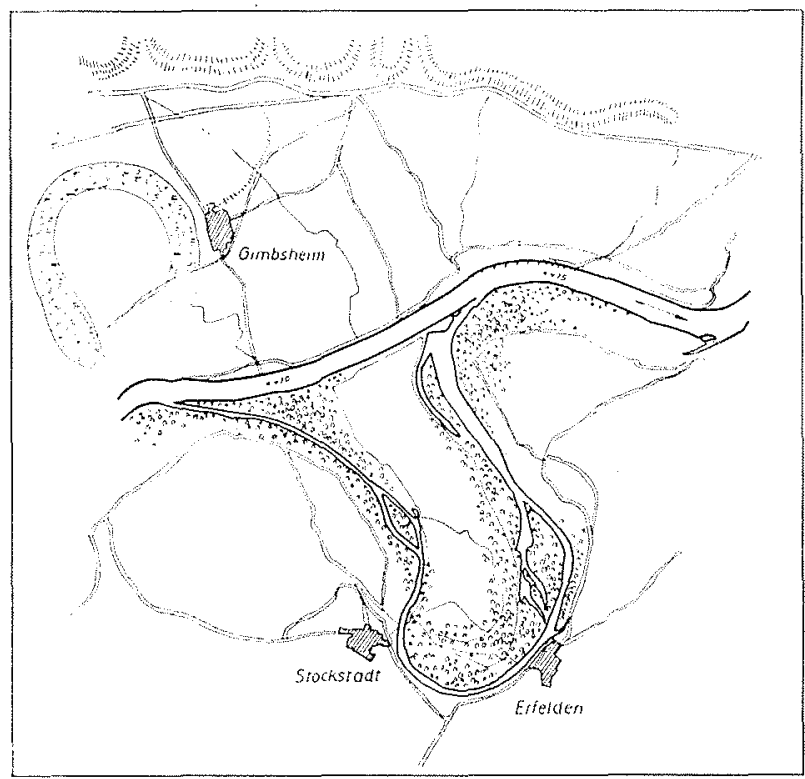

Fic, 11

Coupure au Geyer. - Etat actuel

tudinaux et des épis pour améliorer les conditions d'écoulement en de nombreux endroits, (fig. 12). Près de Worms en 1850-1853, on ferma une «baie» sur la rive gauche du fleuve au moyen d'un ouvrage de guidage, créant ainsi en même temps un bassin portuaire. De plus, au cours des années, on aménagea les parcours situés plus loin en aval par des guideaux et des épis, de manière à fixer le cours du fleuve à sa largeur normale et faisant disparaître ainsi les dépôts solides qui se formaient autrefois sans cesse, ainsi que les ramifications du lit navigable.

On réunit en une seule, les deux îles de 《Kisselwörth » et «Sändchen », près de Nackenheim, régularisant ainsi la répartition de débits 
entre les bras principal et secondaire en amont de Nackenheim. Au «seuil de Nackenheim», banc rocheux extrêmement gênant pour la navigation, on obtint une amélioration considérable en travaillant aux explosifs et en implantant des épis le long de la rive droite, fermant en même temps le «Vieux-Rhin de Ginsheim », sans toutefois arriver à éliminer toutes les difficultés de manière définitive.

Tous ces travaux furent exécutés pendant la période 1860-1910, et ont eu pour résultat de procurer la profondeur navigable désirée de $1,70 \mathrm{~m}$ au-dessous de l'E.E., laquelle existe encore partout de nos jours. (L'E.E. ou «étiage équivalent», est la hauteur d'eau fictive au-dessous de laquelle la cote réelle ne tombe que pendant, en moyenne, 20 jours de l'année). Notons enfin sur ce parcours, un aménagement complémentaire, qui fut réalisé sur une boucle étroite à l'aval de Gernsheim, au «Schwarzer Ort», en 1932. Là, on améliora les conditions d'écoülement, d'une part par des travaux d'excavation, et d'autre part en avançant la rive droite dans le fleuve, permettant ainsi à de longs convois de se croiser sans risque, et empêchant surtout la formation d'accumulations de glaces telles qu'elles se produisaient si souvent autrefois.

Le parcours du Rheingau entre Mayence et Bingen est caractérisé par sa largeur relativement grande et sa faible pente, toutes deux provoquées par l'effet de retenue vers l'amont produit par un seuil rocheux sis en travers du fleuve à l'aval de Bingen, la fameuse «Barre du Trou de Bingen ». De nombreuses îles, entre lesquelles les différents bras du fleuve changeaient constamment de largeur et de profondeur en raison des matériaux qu'ils déposaient, caractérisaient autrefois ce parcours. Les travaux de régularisation réalisés à cet endroit pendant le $\mathrm{XIX}^{\mathrm{e}}$ siècle avaient pour objet d'éliminer la plupart de ces bras et d'établir un chenal navigable suffisamment large et profond. Certaines îles furent jointes aux rives en comblant des bras de dérivation du fleuve, et on en élimina d'autres par des travaux de terrassement, ou bien on les relia entre elles par des ouvrages longitudinaux afin d'éviter des courants transversaux et l'envasement du bras principal. En donnant des formes convenables aux têtes amont des îles, on parvint à répartir convenablement l'écoulement entre les différents bras du fleuve. La construction de revêtements des rives et d'ouvrages de guidage au niveau des moyennes eaux avait pour objet de réunir les moyennes eaux de telle manière que, - même en période d'étiage -, il reste toujours une profondeur navigable suffisante sans toutefois porter un préjudice inadmissible aux débits s'écoulant en période de crue.

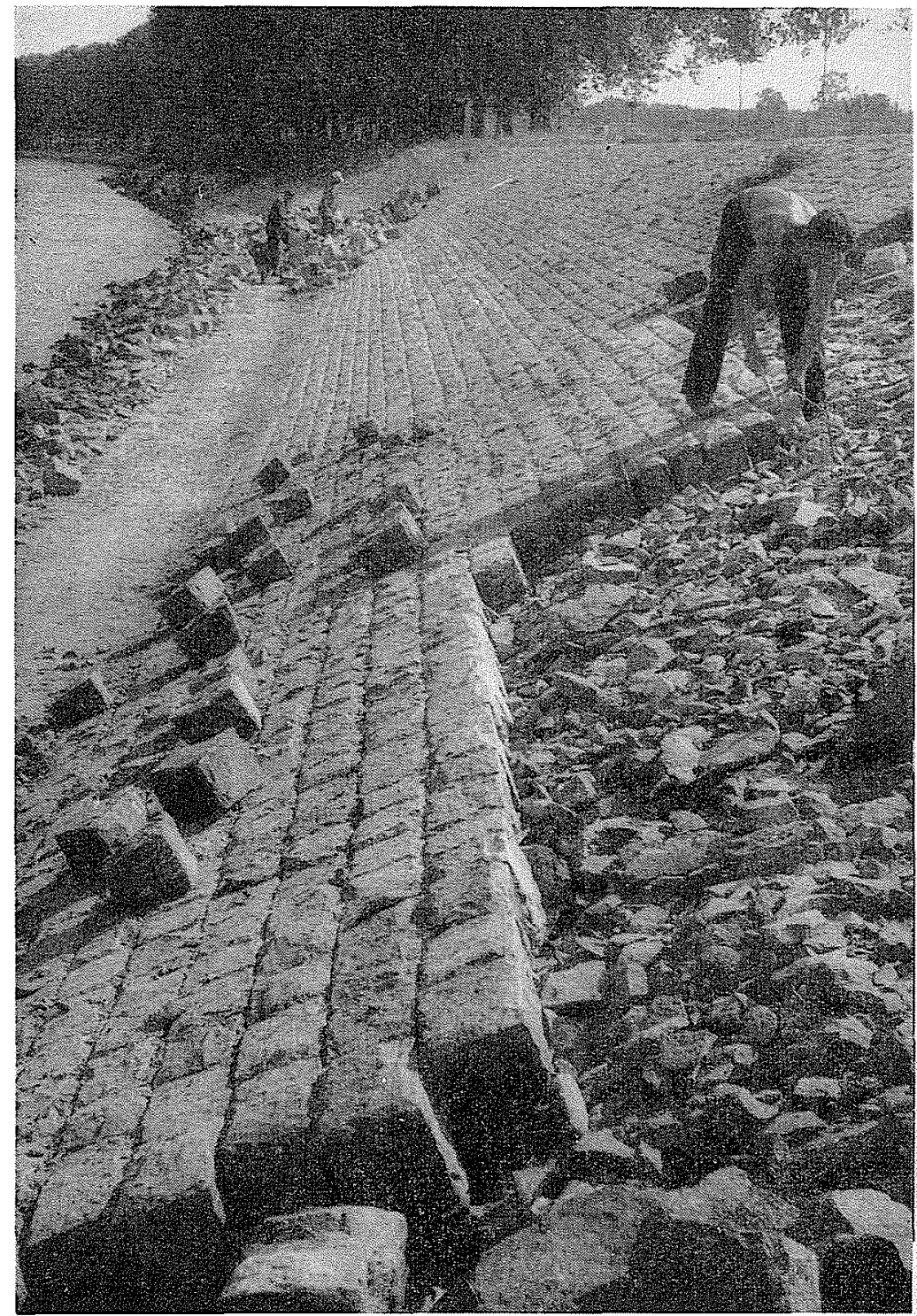

Fic. 12

Réalisation d'un perré de berge

L'étude et la réalisation de ces travaux furent rendues plus difficiles par le fait qu'il fallait d'abord obtenir la permission des deux Etats intéressés (Hesse et Nassau, ce dernier remplacé plus tard par la Prusse) et que leurs intérêts n'étaient pas toujours en accord. Outre les intérêts portant sur le trafic et la circulation, ceux de l'agriculture surtout, et en particulier de la viticulture, entraient considérablement en jeu. Néanmoins la Prusse et la Hesse définirent finalement les bases de l'aménagement au moyen d'une convention signée en 1884. La plupart des travaux déjà entrepris, bien que ne se conformant pas toujours aux mêmes points de vue, furent terminés avant la fin du siècle. Or, la 


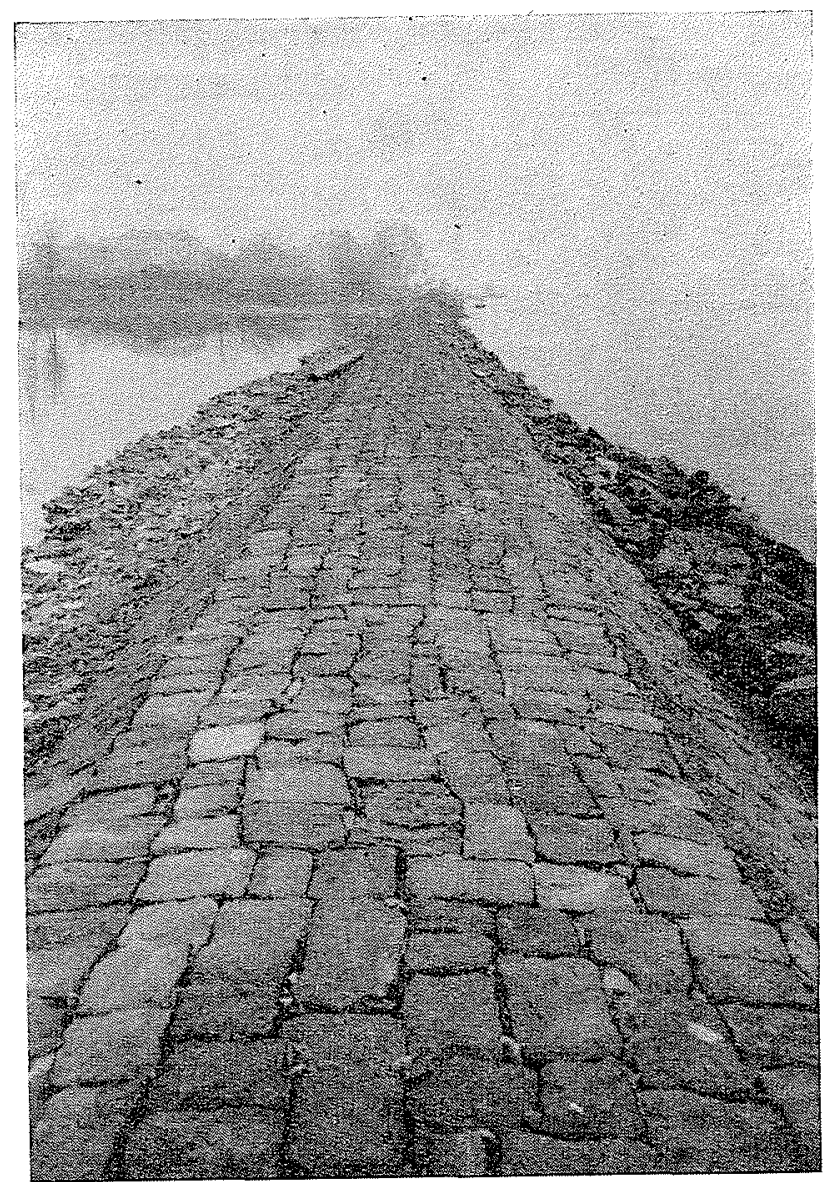

Fic. 13

Ouvrage longitudinal sur le parcours du Rheingau

convention de 1884 interdisait de diminuer l'aire du plan d'eau, ceci dans l'intérêt de la viticulture, et tous projets d'aménagement devaient tenir compte de ce point de vue. On ne construisit donc sur ce parcours aucun épi, évitant ainsi l'atterrissement se produisant normalement sur les champs situés entre de tels ouvrages, et on se borna à ne prévoir que des ouvrages longitudinaux (fig. 13); bien que ceux-ci séparent inévitablement des étendues d'eau du cours principal en temps d'étiage, par contre, ils ne sont pas sujets à l'atterrissement et restent par conséquent toujours recouverts par les eaux. La figure 14 montre un de ces systèmes d'ouvragesguides, situé au branchement entre la «Grande Giess» et la «Petite Giess». Notons enfin les plus importants de ces aménagements :

1884: Régularisation des berges à la Maaraue, en aval de Mayence.

1885-1887 : Implantation d'épis à Niederwalluf et Geisenheim.

Aménagement des berges sur le parcours Eltville-Oestrich.

Fermeture de la «baie» et du vieux bras a Hattenheim. Travaux de régularisation à la «Westfälische Au», et dragages sur la Grande Giess et la Petite Giess.

Réalisation de l'ouvrage parallèle en amont de Freiweinheim.

Elimination de la «Winkeler $A u »$ et du

«Winkeler Wörth».

Aménagement de la «baie» de Mittelheim.

Aménagement du chenal navigable à Rüdesheim et Kempten.

1888-1890 : Construction d'un ouvrage de guidage à Kastel, et d'ouvrages parallèles dans le bras de Mombach, et à Freiweinheim.

Pendant les années 1892-1896, on termina les travaux d'aménagement du chenal navigable de Rüdesheim et de Kempten; par ailleurs, à Bingen, on régularisa une «baie» riveraine au

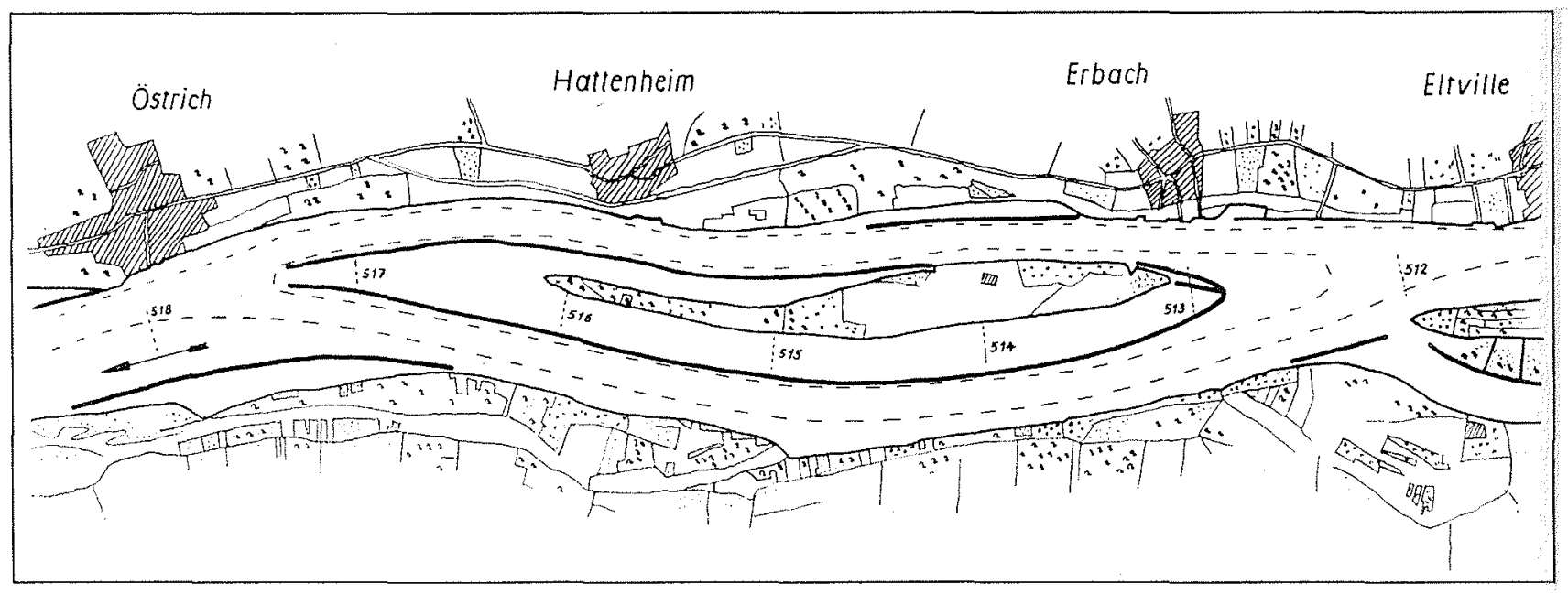

FIG. 14. - Régularisation par des guideaux. - Parcours du Rheingau 
Fig. 15

Vue acrienne du "Trou de Bingen» et du

confluent du Nahe.

$$
\begin{array}{r}
\text { (Photo } \\
\text { Aéro-Exploration, } \\
\text { Aéroport } \\
\text { de Franctort.) }
\end{array}
$$

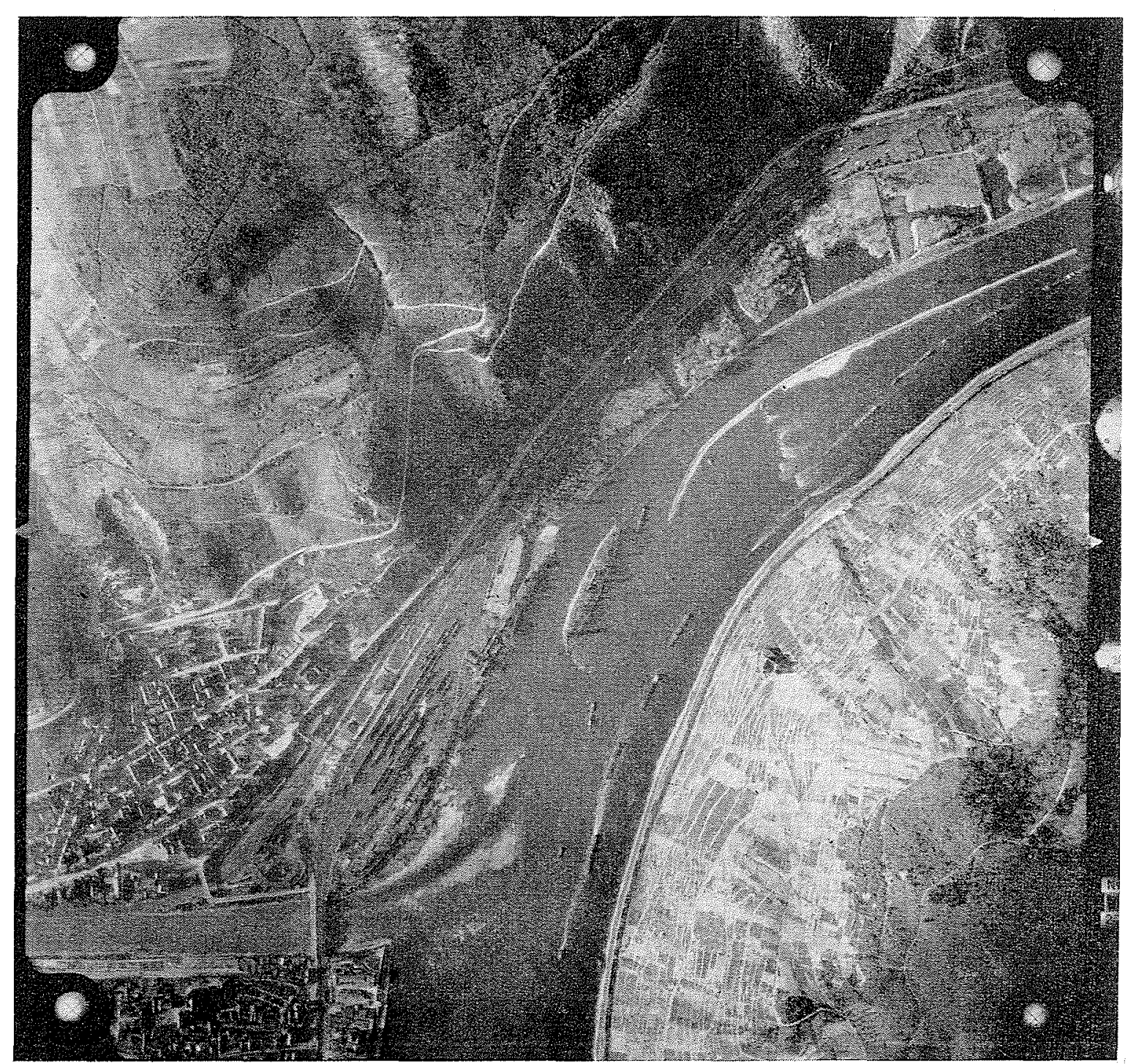

moyen d'une digue de remblais renforcée, (comme sur le parcours en amont de Worms, voir plus haut), créant simultanément un port de refuge et de tête de ligne.

Enfin, des travaux de régularisation complémentaires furent encore réalisés en 1903 et en 1904.

La construction de seuils de fond, des dragages, et des améliorations apportées à quelquesuns des guideaux constituèrent enfin l'achèvement de cet important programme d'aménagement qui se justifie encore entièrement de nos jours.

Il garantit à la navigation la possibilité de maintenir un trafic de bateaux chargés à plein pendant la plupart de l'année, malgré les quelques difficultés occasionnées encore aujourd'hui en période d'étiage par la présence de bancs de sable, qui ne peuvent toutefois être supprimés qu'en draguant.
A l'aval de Bingen, le Rhin a fait une trouée d'environ $50 \mathrm{~km}$ de long à travers le massif schisteux de la Haute-Rhénanie, Sur ce parcours, entre Bingen et St. Goar, le fleuve se caractérise par des berges escarpées, un fond rocheux très accidenté parsemé de nombreux blocs individuels, des boucles serrées et renfermées entre les hautes berges rocheuses. De là jusqu'au confluent de la Moselle à Coblence, la vallée devient un peu plus large, bien que l'on ne puisse encore considérer ce parcours autrement que comme une partie du parcours montagneux.

La barre du «Trou de Bingen» est le plus dangereux obstacle pour la navigation sur tout le cours du Rhin, (voir fig. 15). Les essais visant à l'amélioration des conditions à cet endroit remontent à plusieurs siècles; au $\mathrm{Xrv}^{\mathrm{e}}$ siècle ce fut surtout la Maison commerciale von Stockum, de Francfort, qui chercha à y assurer un chenal navigable toujours utilisable. Toutefois, ce ne 


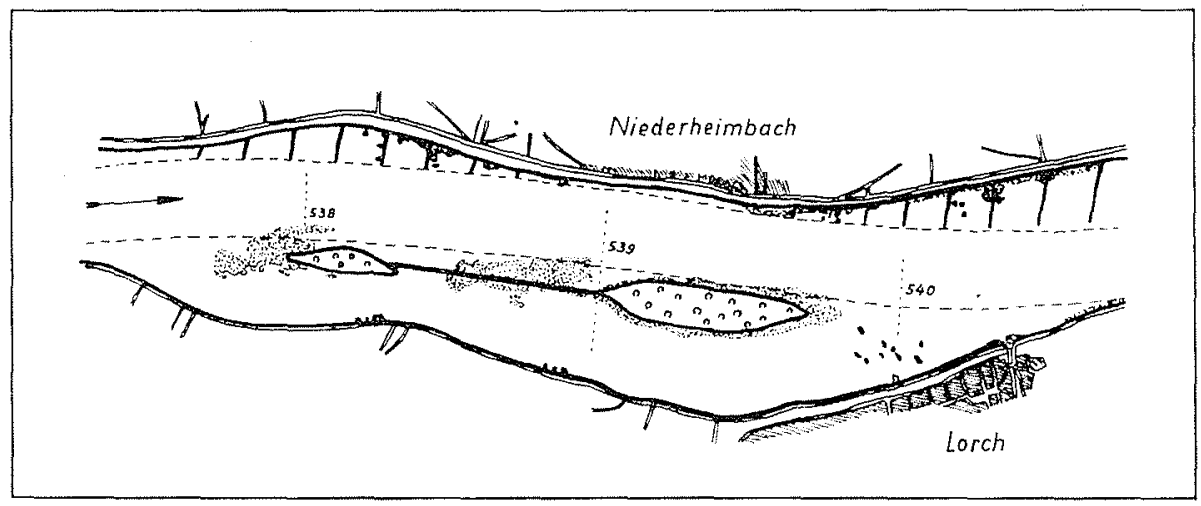

Fig. 16

Liaison des deux îles à Loreh par un guideau fut qu'en 1814, lorsque le Service prussien d'administration des Eaux fut chargé de la gestion de ce parcours, et que les moyens techniques nécessaires étaient disponibles, que l'on parvint à élargir la passe navigable près de la rive droite; en travaillanit aux explosifs, cette passe, large jusqu'alors de seulement 7 à 9 mètres, fut amenée à 23 mètres, ceci pour une profondeur navigable de $1,44 \mathrm{~m}$ par rapport à la cote moyenne des basses eaux. Après l'an 1861, on entreprit l'aménagement d'un deuxième chenal de navigation, à laquelle fin on remblaya un guideau d'environ $1 \mathrm{~km}$ de long sur le côté gauche de la barre, et l'on donna à la cuvette du chenal entre ce guideau et l'île de la «Tour des Souris », une forme convenable, afin de pouvoir répartir sur toute la longueur de ce chenal, la chute entièrement concentrée sur une très courte distance au "Trou de Bingen». Ce chenal est emprunté surtout par les bateaux naviguant d'amont en aval, alors que les convois remontant le fleuve passent pour la plupart par le chenal du «Trou». En aval de la «Tour des Souris», on implanta dans la largeur excédenlaire du fleuve, côté rive gauche, un système d'épis dont les extrémités étaient reliées par un guideau.

L'accroissement continu du trafic, ainsi que l'augmentation progressive des tonnages des bateaux utilisant le fleuve étaient tels que l'on étudia, dès la première décennie du présent siècle, les possibilités d'aménager encore davantage ce passage difficile du «Trou de Bingen». L'objet de ces études était surtout de chercher à définir les différents moyens d'améliorer la section existante, mème en installant des systèmes d'écluses si nécessaire. Ces études ne produisirent aucun résultat satisfaisant. On les reprit après la fin de la première guerre mondiale, et des travaux d'amélioration furent commencés dans la deuxième section en 1925. Il s'agissait de supprimer des pointes rocheuses, d'aménager des seuils de fond, et de diminuer la largeur du fleuve, travaux qui furent achevés en 1932. Les bateaux disposent actuellement au «Trou de Bingen» de deux chenaux navigables, dans lesquels la profondeur désirée de $1,70 \mathrm{~m}$ au-dessous de I'E.E. est toujours disponible.

Notons parmi les ouvrages de régularisation nécessaires sur le parcours Assmannshausen St. Goar, les suivants : Un important groupement d'épis en aval de 'Trechtingshausen et à Niederheimbach; un ouvrage de guidage reliant les deux îles à Lorch (voir fig. 16) afin d'éviter des courants transversaux défavorables; les travaux d'aménagement entre Bacharach et Kaub, où l'on créa, en aménageant des groupements d'épis et des digues de séparation et en travaillant aux explosifs, les deux chenaux navigables nommés « das wilde Gefähr» et « das Kauber Wasser », établissant ainsi des conditions tolérables sur ce parcours qui est le plus dangereux après le «Trou de Bingen ». Entre Kaub et St. Goar, à l'aval, il suffit de supprimer quelques rochers gênant la navigation. Un important élargissement, avec un affaiblissement correspondant de la pente du profil en aval de la Lorelei donnait lieu à des envasements et des divisions du fleuve, et il fallut le supprimer au moyen d'épis disposés le long de la rive gauche et d'un guideau en rive droite. On aménagea simultanément un port de refuge et de sécurité sur cette rive droite en élevant ce guideau audessus de la cote de crue; or il est évident qu'un tel port, situé juste à l'entrée du parcours rocheux présente un intérêt tout particulier.

Entre St. Goar et Coblence, la vallée s'élargit, les berges se retirent et deviennent moins escarpées. Le lit du fleuve est constitué de graviers, et sauf en trois endroits (Wellmich, Ehrenthaler Werth, et à l'aval d'Osterspay), aucun aménagement important n'a été nécessaire. En face de Wellmich, un groupe d'épis a été installé, dont on relia les extrémités par un guideau. On augmenta plus tard la hauteur de ce dernier (entre les années 1936-1940), en draguant également la «baie» derrière l'ouvrage, ce qui créa le port de refuge «Am Hund». Au 
"Ehrenthaler Werth» on délimita deux chenaux navigables, l'un de $150 \mathrm{~m}$ de large et l'autre de $100 \mathrm{~m}$, en implantant des épis et en y apportant un revêtement d'environ $1,5 \mathrm{~km}$ de long. Sur la boucle étroite en aval d'Osterspay, où le fleuve s'était élargi considérablement à cause de l'érosion importante et incessante de la rive droite, on implanta un ouvrage de séparation de $1,7 \mathrm{~km}$ de long, créant ainsi deux chenaux navigables; celui de gauche fut encore délimité par un ouvrage longitudinal et des épis sur la rive gauche.

A l'aval de Coblence, le Rhin traverse le large bassin de Neuwied; les montagnes s'approchent de nouveau du fleuve à Andernach et Leutesdorf, ne s'en éloignant plus jusqu'à Bonn. De là le Rhin devient un fleuve de plaine. Son débit est considérablement augmenté par les apports du Lahn et de la Moselle, et bien que sa pente soit encore très irrégulière, elle l'est toutefois moins que sur: le parcours rocheux. Le soubassement est constitué de graviers reposant souvent sur une fondation très ferme. C'était surtout à cause des îles et des bancs de graviers qu'il devint nécessaire de réaliser des aménagements pour améliorer la navigation sur le parcours entre Coblence et Cologne. On relia les bancs de graviers au voisinage de la rive gauche avec celle-ci par des épis, ce qui établit des conditions stables pour la navigation dans le bras gauche principal du fleuve; on exécuta également des travaux semblables au «Weissenthurmer Werth», au «Hammersteiner Werth», au «Nonnenwerth», et enfin au "Graswerth» près de Honnef. Toutes ces îles furent fixées par des guideaux, des revêtements et des épis, de façon à stabiliser le chenal principal sur des largeurs et des profondeurs adéquates. L'aménagement et la stabilisation des berges sur tout le parcours se poursuivaient en même temps que ces travaux, par l'installation d'ouvrages de couverture et d'épis. Ainsi, ce fut en appliquant un grand nombre de mesures individuelles que l'on travailla sans cesse à l'amélioration des conditions de navigation pendant la période allant de la fondation du Service prussien de l'Aménagement du Rhin à Coblence en 1851, jusqu’à la première guerre mondiale; on dispose par conséquent aujourd'hui, sur tout le parcours CologneCoblence, de la profondeur navigable désirée de 2,10 $\mathrm{m}$ au-dessous de l'E.E., et ce pour des largeurs convenables. De plus, la navigation ne se heurte plus à des difficultés appréciables, même en temps d'étiage; signalons toutefois que l'entretien des ouvrages entraine des dépenses considérables.

Il reste encore à évoquer la création des ports de refuge et de sécurité aménagés dans le cadro de ces travaux à Brohl et à Oberwinter : à cette fin, on $y$ construisit, comme on l'avait fait à l'aval de la Lorelei, des digues longitudinales et l'on aménagea convenablement les «baies » riveraines à ces endroits. Les figures 17 et 18 montrent des aspects caractéristiques de ces travaux.

Sur le parcours entre Cologne et DuisburgRuhrort, le Rhin changeait autrefois souvent son cours, dans les régions plates de la basse-plaine de l'Allemagne du Nord. A peu près comme dans la basse-plaine de la Rhénanie du Nord, il décrivait d'immenses méandres, perçant les étroites bandes de terre entre ces boucles en période de crue ou de gel et laissant des bras morts qui, loutefois, s'atterrissaient par la suite. De nombreuses îles parsemaient son cours, le divisant et gênant la navigation. La zone inondable près de Cologne est large d'environ $1 \mathrm{~km}$, et de 8 à $10 \mathrm{~km}$ dans la région de Duisburg-Ruhrort. Cette zone fut peu à peu protégée contre les inondations par des digues, sur de longs parcours, et l'on supprima les îles ou bien on les relia aux rives, de sorte qu'il existe actuellement un lit bien défini. Afin d'assurer les largeurs et les profondeurs nécessaires dans ce lit, il fallut, ici comme ailleurs, régulariser des avancements des rives, éliminer des bancs de sable au centro du fleuve et stabiliser les berges. On employa à cette fin des revêtements, des digues longitudinales et des épis classiques avec beaucoup de succès.

Nous sortirions du cadre des présentes considérations si nous examinions en détail toutes les mesures prises. Notons seulement qu'en fonction de l'industrialisation toujours plus poussée de

\section{FIG. 17}

Port de refuge et de sécurité à Brohi

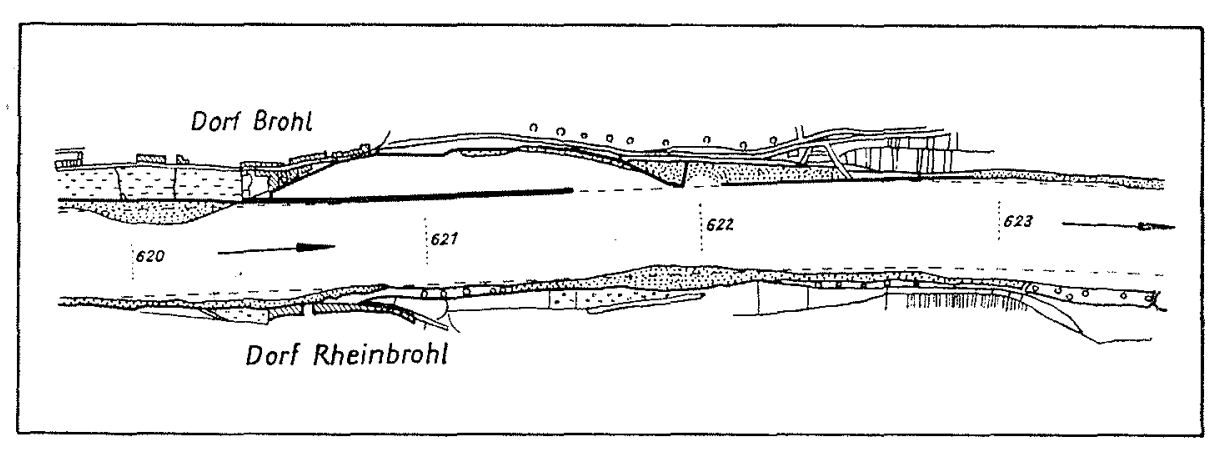




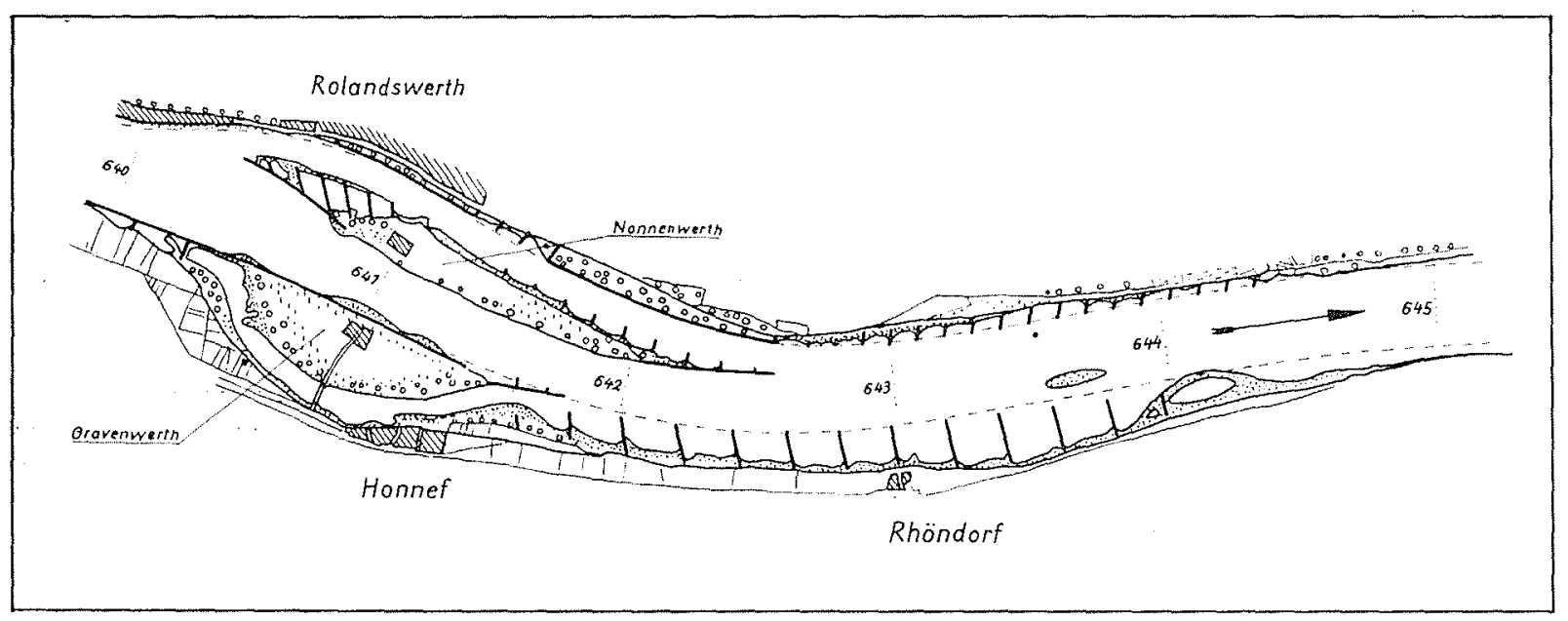

Frg. 18. - Ouvages de régularisation aux iles de Nonnenwerth ef Gravenwerth

ces régions, les aménagèments réalisés dans la région de Cologne et de Düsseldorf --- mais également dans d'autres endroits - - afin de faciliter la navigation directe, allèrent de pair avec d'autres travaux : installation d'un port de tête de ligne pour le trafic local, de quais et d'installations industrielles. Toutes ces mesures ont profondément changé l'aspect original du paysage du Rhin.

Deux aménagements très importants réallses au cours des années récentes méritent une mention toute particulière :

Dans une courbe très serrée près de Düsseldorf, le lit comportait d'immenses profondeurs locales provoquées par l'affouillement, profondeurs qu'il fallait combler à tout prix. Ayant déli.

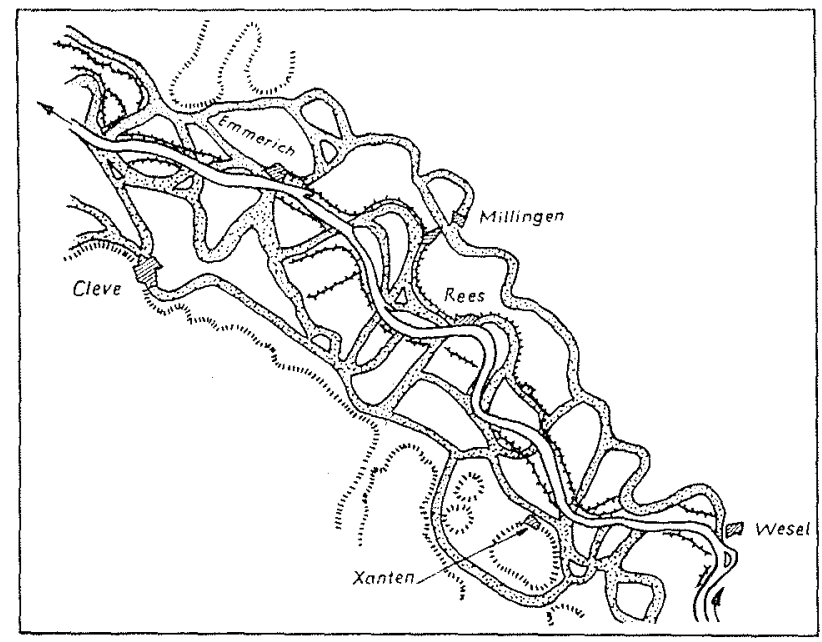

FIG. 19

Changements de cours dans la région du Rhin Infërieur, i la suite de crues et de gels béré, et étudié le problème en considérant ses différents aspects, dans le but de choisir le genre d'ouvage qui conviendrait le mieux, on décida finalement de combler les creux affouillés et de recouvrir le tout avec des pierres très lourdes. Cette solution s'est montrée entièrement satis. faisante jusqu'à présent.

A l'amont de Düsseldorf, une barre de quarlzite traverse tout le fleuve, de laquelle se détachent sans cesse des pierres qui constituent un risque pour la navigation. On a déblayé ces pierres en employant des puits de plongée, éliminant ainsi ce danger dans une grande mesure, mais il reste néanmoins nécessaire de toujours maintenir un contrôle sévère à cet endroit.

Plus encore que dans la zone entre Cologne el Duisburg-Ruhrort, le Rhin était autrefois retourné à l'état sauvage sur le parcours allant du confluent de la Ruhr jusqu'à la frontière germano-néerlandaise (fig. 19). D'importants changements de lit provoqués soit par les crues, soit par les glaces, se produisaient sans cesse, détruisant des localités entières qui devaient par la suite être reconstruites autre part. Les mesures locales prises he pouvaient présenter de solution permanente, étant donné qu'elles n'étaient jamais appliquées systématiquement, et pouvaient même provoquer des dégâts sur d'autres parties du parcours. De plus, l'éparpillement des pouvoirs des différentes administrations locales fut en grande partie responsable du manque de tout programme coordonné. L'aménagement systématique du parcours ne devait se faire que sous les règnes de Frédéric II et de son successeur, où l'on élimina des îles nuisibles, créa un tracé d'écoulement uniforme, le stabilisant par des revêtements et des épis convenablement aménagés. L'organisation et l'exécution de ces travaux furent, pour cette époque, une 
performance remarquable, une réalisation qui sera toujours liée au nom de l'éminent hydraulicien fluvial que fut Wiebeking. Toutefois les différents ouvrages ne furent plus entretenus pendant les guerres napoléoniennes, et presque tous tombèrent peu à peu en ruines. En 1816, l'administration des eaux dans le territoire prussien fut confiée aux gouvernements nouvellement constitués, qui commencèrent la reconstruction des ouvrages. En 1851, on chargea Nobiling, qui dirigeait déjà depuis 1843 tous les travaux d'aménagement dans la région administrative de Düsseldorf, de l'administration et de l'exécution des travaux analogues sur tout le parcours prussien du Rhin; on créa à cette fin le Service d'Aménagement du Rhin, et on nomma Nobiling directeur de cet organisme.

Il s'agissait surtout, dans l'aménagement do cette partie du Rhin-Inférieur, de fixer le cours sur un soubassement constitué de matériaux de faible granulométrie et de le guider de facon à pouvoir garantir l'équilịbre du régime des transports solides, tout en assurant des profondeurs et largeurs adécuates pour la navigation, et en maintenant les frais d'entretien au plus bas niveau possible.

Les systèmes de digues existant à l'époque se prêtaient mal à l'aménagement, étant donné l'alternance de zones étroites et de zones inondables étendues entre les digues; or, alors que ces larges lits inondables conviennent très bien en tant que «zones de polders» pour l'écrêtement des crues, par contre les dépôts de matériaux solides qui s'y produisent donnent lieu à une attaque plus considérable du lit dans le cours d'écoulement proprement dit, déséquilibrant ainsi le régime des transports solides.

Il fallait donc que, sur le Rhin-Inférieur en particulier, les aménagements se fassent de pair avec l'amélioration des systèmes de digues, bien qu'il ne fût pas toujour's possible d'effectuer les déplacements nécessaires de ces digues, à cause des localités et des installations industrielles existant déjà sur les lieux. Jusqu'à 1850, les travaux de régularisation se limitèrent principalement à la mise en place de revêtements sur les berges et au renforcement des chemins de halage; ce ne fut qu'après ces travaux que l'on attaqua la régularisation proprement dite, cherchant à assurer les largeurs et profondeurs nécessaires du lit au moyen d'épis et de seuils de fond.

Les travaux principaux furent effectués penclant les années 1880-1900, réussissant à éliminer en grande mesure les ramifications du cours navigable formées par des bancs de sable et de gravier, à aménager convenablement des élargissements du lit qui étalaient le chenal navigable, et à créer le lit actuel qui traverse la hasse- plaine du Rhin en traçant des méandres déliés le long de son cours.

Bien que le gros de ce travail de régularisation fût achevé à la fin du dernier siècle, des travaux courants complémentaires devinrent toutefois encore nécessaires au cours des décennies suivantes, afin de conserver et d'améliorer ce qui avait été réalisé. C'est ainsi qu'en 1908/09 il fallut exhausser des creux au moyen d'un groupe de seuils de fond près d'Emmerich, et maintenir des profondeurs adéquates à d'autres endroits par des dragages répétés.

L'érosion, toujours en progrès sur le RhinInférieur, reste encore une source d'inquiétudes, provoquée qu'elle est d'une part par l'insuffisance des apports de matériaux solides provenant de l'amont, et d'autre part, comme nous l'avons signalé plus haut, par la largeur excessive des zones inondables, sur lesquelles les matériaux fins se déposent même lors d'une faible inondation et les exhaussent, de sorte que toute crue importante attaque alor's inévitablement le lit du fleuve. La solution idéale serait de prévoir un système de digues uniforme, avec des espaces égaux entre les digues et les zones inondables plates sur les berges, calées à une cote d'environ $1,0 \mathrm{~m}$ au-dessus de la cote des moyennes eaux; la transition de ces zones aux digues aurait un profil en faible pente, permettant ainsi aux eaux de crue de s'écouler sans danger entre les digues. Toutefois, étant donné les événements au cours de l'histoire du fleuve, cette solution n'est plus entièrement réalisable depuis longtemps.

Grâce à la collaboration entre les Services responsables des digues d'une part, et d'autre part les Services des eaux et de la navigation, des succès remarquables ont néanmoins pu être enregistrés en renforçant des digues sur de longs parcours, ainsi qu'en en déplaçant et en en aménageant d'autres. Par exemple, on a pu réaliser un profil très favorable à l'écoulement des eaux de crue lors des travaux de renforcement. et de reconstruction de la digue Berl-Ossenburs effectués en 1936/38, en èxtrayant de la zone inondable les matériaux de construction pour la digue et arasant ainsi la zone inondable à la cote $1,0 \mathrm{~m}$ an-dessus de la cote des moyennes eaux. Cette collaboration entre les Services intéressés a également rendu possibles à plusieurs autres endroits des améliorations notables, portant sur l'écoulement des eaux de crue et la protection des terres. Dans la période entre les deux guerres mondiales, le Service d'aménagement du Rhin a étudié un projet général de régularisation des crues, auquel se conformeront désormais tous travaux d'aménagement et de constructions de dignes. Les importantes quantités de gravier requises pour les travaux de construction, tant 
dans la région industrielle $d u$ Rhin-Inférieur allemand qu'aux Pays-Bas, ont provoqué l'exploitation très poussée des ressources en ce matériau dans les zones inondables; toutefois, cette exploitation, ainsi que tout remblayage des surfaces exploitées, sont réglementés par une législation corrélative, assurant en définitive le maintien des cotes requises pour l'écoulement régularisé des eaux de crue sur les zones intéressées.

C'est surtout depuis le début du XIX siècle que l'on a réalisé les travaux les plus importants dans le domaine des aménagements fluviaux, de la mise en valeur des terres et de la navigation. Sans ces travaux, le trafic que l'on connaît aujourd'hui sur le parcours entre la frontière germano-néerlandaise et la région en amont de Bâle (108 millions de tonnes transportées, et 23,4 milliards de tonnes/kilomètres en 1957) n'aurait jamais été possible; de plus, les installations industrielles le long des deux rives du Rhin n'auraient guère pu évoluer comme elles l'ont fait, si la création d'une excellente voie de navigation ne s'était accompagnée de mesures pour la protection des zones riveraines contre les caprices du fleuve. S'il existe actuellement les profondeurs navigables nécessaires de $2,5 \mathrm{~m}$ audessous de l'E.E. en aval de Cologne, de $2,10 \mathrm{~m}$ entre Cologne et St. Goar, et de $1,70 \mathrm{~m}$ à l'amont de St. Goar, si les largeurs disponibles sont généralement suffisantes, et si les dégâts causés par les crues et les glaces ne risquent plus de devenir catastrophiques, ce n'est que grâce à la vision et la perspicacité des anciens spécialistes de la régularisation fluviale, qui en donnant au fleuve l'aspect que nous connaissons aujourd'hui, méritent d'autant plus notre reconnaissance.

On peut considérer en général l'aménagement du fleuve comme terminé. Aujourd'hui et dans l'avenir, il ne reste plus, en général, qu’à réaliser des améliorations locales pour assurer encore davantage le rendement du Rhin en tant que voie de communication, et d'essayer de réduire autant que possible les restrictions portant sur le déchargement des bateaux en période d'étiage.

En considérant de ces points de vue les divers parcours partiels du fleuve dont nous venons de décrire les aménagements réalisés dans le passé, nous pouvons constater, pour le parcours entre la frontière franco-allemande et Mannheim, les faits suivants :

Dans les zones intéressées par les aménagements des basses eaux en amont de Sondernheim, la profondeur voulue de $1,70 \mathrm{~m}$ au-dessous de l'E.E. a été pratiquement créée. Les seuls travaux restant à faire sur ce parcours seraient quelques améliorations locales effectuées dans le cadre des travaux d'entretien normaux, sous la forme d'un aplanissement des berges ayant pour objet de créer un régime d'écoulement plus permanent et tranquille, et permettant d'envisager une meilleure configuration du lit d'étiage. En omettant par endroits des groupes d'épis lors de limplantation des ouvrages de régularisation des basses eaux effectués dans le passé, on a laissé de nombreux endroits dégagés permettant aux bateaux de virer. Toutefois, non seulement ces emplacements ont perdu toute utilité pour la navigation au cours des années, mais encore ils ont eu une influence très défavorable sur la régularisation du fleuve. Le manque d'épis à ces endroits interrompt le caractère permanent de l'écoulement dans le lit d'étiage, donnant lieu à des dépôts localisés de matériaux solides. Les zones de virage s'ensablaient également sans cesse, rendant nécessaire des dragages fréquents; en outre, c'était justement pendant les périodes d'étiage que leurs possibilités d'utilisation devenaient problématiques. On en abandonna donc peu à peu la plupart, et on y aménagea des ouvrages de régularisation. Les deux dernières de ces zones, situées l'une sur la rive droite près de Karlsrube, et l'autre sur la rive gauche à Maxau, ont été éliminées au cours des dix dernières années en y construisant les épis faisant défaut, ce qui a enfin eu raison des difficultés provoquées par l'ensablement incessant qui s'y produisait auparavant.

Le parcours entre Sondernheim et Mannheim offre un contraste frappant avec celui à l'amont de Sondernheim, car les seuls travaux effectués jusqu'à présent ont été des mesures pour la régularisation partielle du lit d'étiage (voir plan de situation -, fig. 15).

Il existe donc encore sur ce parcours des profondeurs inadéquates, ce qui fait que les conditions pour la navigation sont en partie moins favorables qu'à l'amont pendant les périodes d'étiage. Les possibilités d'améliorer cet état de choses sont actuellement à l'étude, et les résultats déjà obtenus laissent prévoir les aménagements complémentaires suivants :

\section{Troncon PARTIEL de SONDERnHEIM :}

En période d'étiage, la largeur excédentaire du fleuve et l'absence d'un guidage convenable entraînent la division du fleuve en deux chenaux séparés l'un de l'autre par un banc médian assez long.

En construisant des épis et des guideaux, et en aplanissant les berges, il sera possible d'éliminer ce banc médian et de créer un seul lit bien défini.

\section{Tronçon partiel Germersheim-Rheinhausen:}

Les groupes d'épis présentent sur ce parcour's des lacunes assez importantes. Etant donné la largeur excédentaire du fleuve, il se produit de temps en temps des dépôts de matériaux solides dans le chenal navigable, suivant l'importance des débits solides. Des travaux de régularisation 
complémentaires seraient nécessaires pour assurer des conditions permanentes et adéquates dans le lit navigable.

\section{Troncon partiel a la courbe de SpIRE :}

Dans les courbes étroites à Altlussheim et Spire, la courbure du lit de correction est si défavorable par rapport à l'alignement droit, que des trous profonds se forment dans ces courbes, en donnant lieu à une érosion considérable des berges; en outre, la transition des courbes à

\section{Troncon partiel Sptre-Ketsch :}

Il se trouve sur les deux rives, à la sortie de la courbe de Spire, des banes d'alluvions qui, bien qu'ils ne se reforment pas toujours audevant des travaux de régularisation des basses eaux comme en amont de Sondernheim, changent néanmoins sans cesse de position, si bien que le thalweg «oscille» constamment dans le lit corrigé. On pourra stabiliser ce thalweg par l'implantation judicieuse d'épis et en aplanissant les berges.

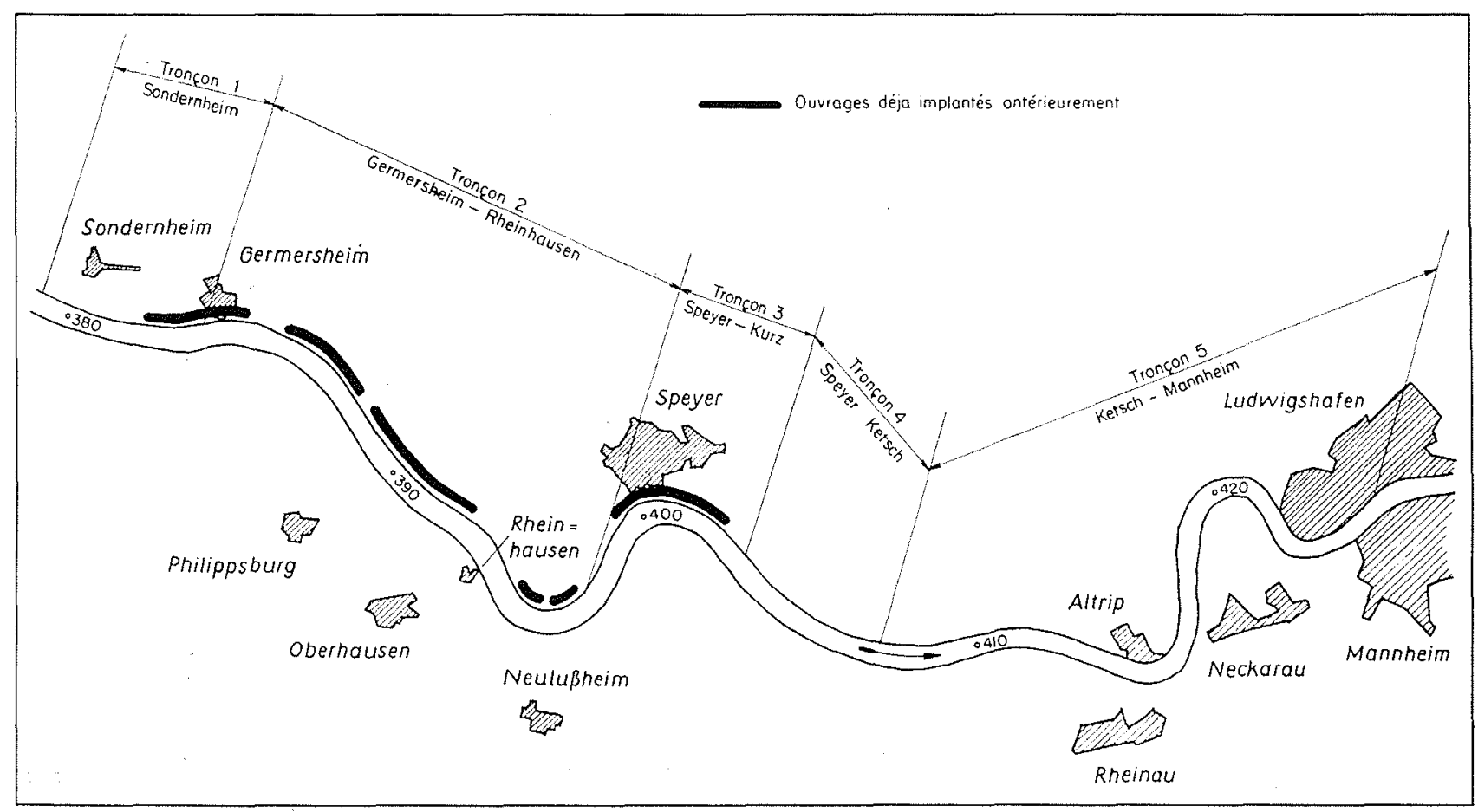

Fig. 20. - Régularisations partielles sur le parcours Sondernheim-Mannheim

l'alignement droit les séparant change tout le temps de position, étant si brusque qu'elle provoque de temps en temps des modifications de la profondeur du chenal navigable. On a par conséquent demandé à l'Institut Fédéral d'Hydraulique (Bundesanstalt für Wasserbau) de Karlsruhe de faire des essais sur modèle réduit afin de déterminer les conditions hydrauliques sur ce parcours et de choisir des aménagements convenables, et il apparaît qu'il y aurait ici également intérêt à aménager des épis et des guideaux, à faire des travaux de terrassement et d'aplanissement des rives pour constituer un lit d'étiage sans transitions brusques et offrant à la navigation un chenal permanent.

\section{Tronçon partiel Ketsch-Mannheim :}

En aval de Ketsch; et jusqu'à Mannheim, l'aménagement actuel du fleuve se présente dans son aspect définitif, ne laissant tout au plus à faire que l'aplanissement des berges pour combler des trous localisés dans le lit.

L'objet de toutes les mesures exposées sommairement ci-dessus est d'apporter des améliorations locales et, ce faisant, de créer des conditions uniformes dans le chenal de navigation sur le parcours entier en amont de Mannheim et d'améliorer ainsi l'accès aux ports du RhinSupérieur. Il n'est pas possible d'entreprendre toutes les mesures individuelles simultanément, 
car il faut d'abord observer les effets provoqués par chacune d'entre elles avant de commencer la suivante.

En aval du confluent du Neckar, il ne reste plus avant Mayence, que quelques endroits isolés où il $\mathrm{y}$ aurait avantage à améliorer le chenal navigable. La profondeur navigable de $1,70 \mathrm{~m}$ au-dessous de la cote E.E. existe presque partout, de sorte que les études concernant ce parcours ont trait à la détermination de la possibilité d'y créer également la profondeur de 2,10 m comme à l'aval du parcours rocheux.

La formation irrégulière des berges en aval de Frankenthal a donné lieu aussi bien à des affouillements du lit qu'à des dépôts de matériaux solides; en aval de Worms, et à Schusterwörth, le fleuve est trop large et tend par conséquent a former des bancs de sable dans son chenal navigable. Dans la courbe de Rheindürkheim, le fleuve érode la rive gauche concave d'une façon très marquée, de sorte qu'il s'est formé un chenal profond et étroit. Il faudra certainement entreprendre ici plus tard des travaux de correction, tant dans l'intérêt de la navigation que pour protéger les ouvrages existants; la nature de ces travaux devra toutefois d'abord être déterminée en détail. Les conditions au seuil de Nackenheim nécessitent des études particulièrement soignées. L'abaissement progressif du plan d'eau à l'aval de ce seuil rocheux depuis le début du XIX siècle laisse toujours subsister des difficultés passagères pour la navigation, malgré les aménagements réalisés. D'après les études effectuées depuis, l'érosion semble être en train de diminuer, mais il sera néanmoins nécessaire de poursuivre ces études très soigneusement jusqu'au bout avant que l'on ne puisse déterminer de manière définitive les mesures qui seraient à appliquer à cet endroit.

Il faudra également réaliser les travaux de régularisation par étapes sur le parcours entre Mannheim et Mayence, afin de pouvoir observer comment le fleuve réagit à ces diverses interventions humaines.

La profondeur de 2,10 $\mathrm{m}$ au-dessous de la cote «E.E.» existe presque partout sur le parcours du «Rheingau» entre Mayence et Bingen. Il reste encore quelques difficultés à la hauteur d'Erbach, là où le chenal navigable se divise en deux bras, dénommés «Grosse Giess » et « Kleine Giess». Il sera vraisemblablement difficile de donner à ces deux chenaux cette plus grande profondeur, et on devra plutôt se contenter de laisser l'un d'entre eux dans son état actuel et tâcher de créer la profondeur voulue dans l'autre, en donnant aux ouvrages de partition à la «Westfälische $\mathrm{Au}$ » des formes appropriées. Les études préliminaires sont actuellement en cours de préparation. A l'aval de l'endroit où la
Grande Giess et la Petite Giess se rejoignent (près de Freiweinheim), le Rhin comporte des largeurs excédentaires et en outre, des vitesses d'écoulement très réduites à cause du remous émanant de la barre au «Trou de Bingen». II se produit par consécquent, sur le parcours Freiweinheim-Rüdesheim, justement pendant les périodes d'étiage de longue durée, de nouveaux dépôts de matériaux solides, qui doivent alors ètre enlevés en draguant. On pourrait remédier à ces difficultés en disposant des guideaux et des revêtements à des endroits appropriés, afin de rétrécir davantage le cours du fleuve, mais ce ne sera qu'en étudiant avec beaucoup de soin le régime des courants, en effectuant des mesures aux différents niveaux du plan d'eau, et le cas échéant par des essais sur modèle, que l'on arrivera finalement à établir les bases de projets d'aménagements convenables.

Le parcours entre Bingen et Assmanmshausen reste toujours le plus difficile de tout le Rhin pour la batellerie, et les avaries subies augmentent en nombre en fonction de l'accroissement du trafic. De plus, toute avarie, mème s'il ne s'agit par exemple que de la perte d'une ancre, entraîne le plus souvent la fermeture de l'un des deux chenaux navigables pendant un temps plus ou moins long.

Tout projet envisageant l'amélioration de ces conditions doit tenir compte de deux problèmes : d'une part creer dans les chenaux de navigation actuels une plus grande profondeur $(2,10 \mathrm{~m}$ audessous de l'E.E., au lieu de $1,70 \mathrm{~m}$ ), profondeur recherchée dans le projet d'approfondissement du chenal navigable en amont de St. Goar, et d'autre part de constituer ces chenaux de telle sorte que le risque d'avarie soit réduit, même pour des plans d'eau plus élevés.

La profondeur de $2,10 \mathrm{~m}$ au-dessous de l'E.E. existe déjà sur une certaine largeur dans le chenal, au «Trou de Bingen», et il suffira d'enlever quelques rochers pour l'obtenir également sans difficultés sur toute la largeur de $30 \mathrm{~m}$. La possibilité d'approfondir le deuxième chenal devra être étudiée dans le cadre du projet de transformation de ce chenal, qui devient nécessaire si l'on veut tenir compte des possibilités de réduire le risque d'avarie et d'accélérer le rythme du trafic fluvial. L'entrée amont de ce chenal est mauvaise; or, avec le nombre toujour's plus grand de bateaux automoteurs, ceuxci utilisent de plus en plus ce chenal en remontant le fleuve. Par suite des conditions défavorables existant à l'entrée utilisée par la navigation allant d'amont en aval, des croisements dans ce deuxième chenal deviennent inévitables, provoquant des arrêts et une gêne mutuelle des navigants. Il sera nécessaire d'étudier diffélentes variantes susceptihles d'améliorer les conditions à cet endroit : 


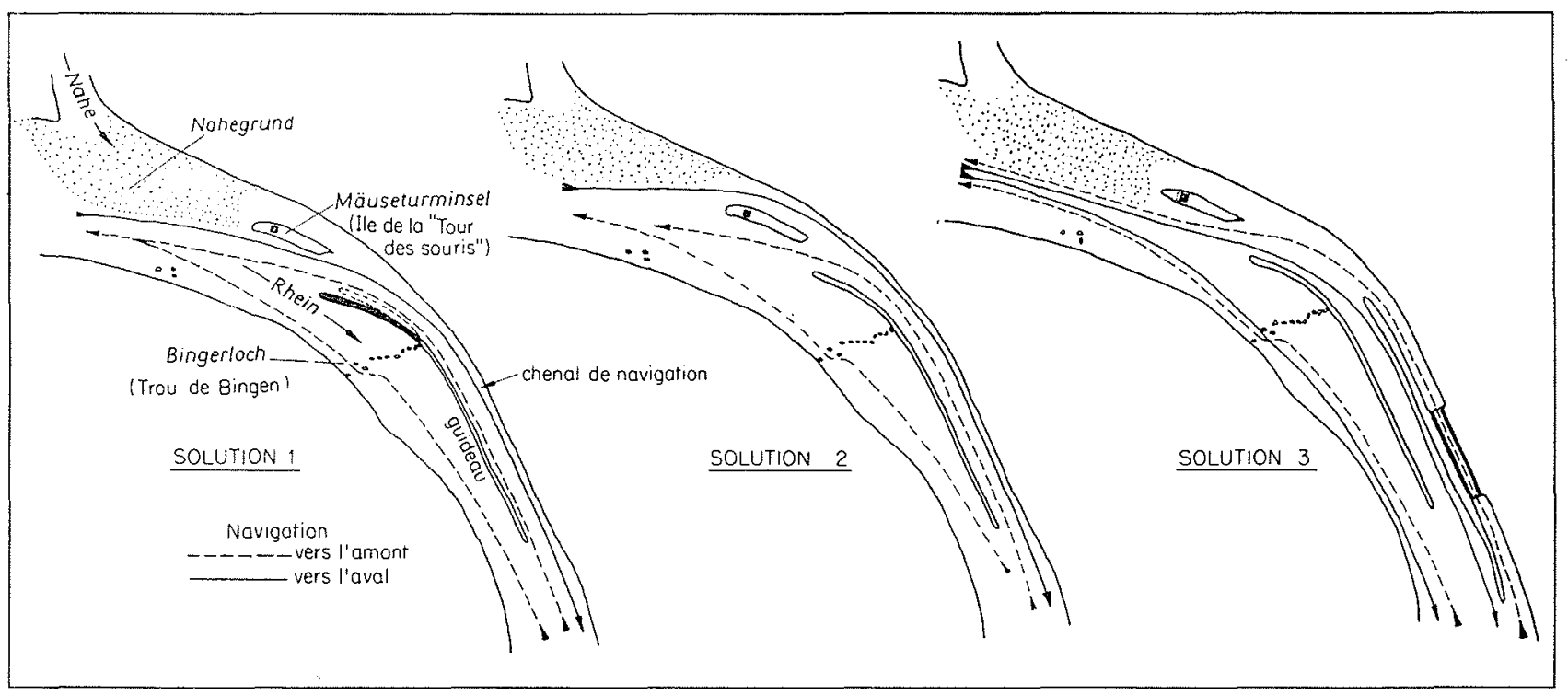

Fra. 21. - Varjantes de projet d'aménagement pour la régula risation du parcours au « Trou de Bingen 》

Variante $n: 1$ : Amélioration de l'entrée amont, et élargissement simultané du chenal navigable, avec l'élimination des profondeurs insuffisantes.

Variante $n^{\circ} 2$ : Création d'un troisième chenal navigable derrière l'île «de la 'Tour des Souris».

Variante $n^{\circ} 3$ : Si les deux solutions précédentes se révélaient impraticables, on construirait une écluse ou un groupe d'écluses. En effet, il $\mathrm{y}$ a suffisamment de place disponible pour implanter un tel ouvrage, mais cette solution entraînerail des dépenses très élevées, et ne serait à envisager par la navigation que comme dernière ressource.

Ces trois solutions sont indiquées par des croquis sur la figure $n^{\circ} 21$.

Il importera surtout dans les études que l'on cflectuera de se rendre compte si le débit du Phin sera toujours suffisant, même en période d'étiage, pour atteindre le but voulu, compte tenu de la réduction correspondante de la section d'écoulement qu'entraînerait par exemple la fermeture des brèches dans la barre sans abaissement du niveau amont et par conséquent du plan d'eau de tout le parcours du « Rheingau $\%$. Les conditions d'écoulement, extrêmement complexes, ne peuvent être déterminées avec suffisamment de précision par le calcul seu], ce qui explique d'ailleurs pourquoi les études effectuées antérieurement n'ont jamais pu aboutir à un projet définitif. On doit arriver à mieux définir ces conditions par ure étude sur modèle en laboratoire, ce qui permet d'escompter au cour's des prochaines années, l'obten- tion de clartés suffisantes sur les aménagements possibles.

La création, dans le parcours rocheux entre Assmannshausen et St. Goar, de la profondeur de navigation de $2,10 \mathrm{~m}$ au-dessous de l'E.E. et l'amélioration des conditions de la navigation aux endroits difficiles nécessiterait encore des aménagements aux deux chenaux de navigation nommés «Wildes Gefähr » et «Kauber Wasser » en amont de Kaub, à la «Rauschelay » en aval de Kaub, ainsi que sur le parcours en aval d'Oberwesel. Bien qu'il s'agisse ici surtout de dérochements, il faudra néanmoins également construire des épis, revêtements et guideaux complémentaires. Il ne suffira pas seulement d'éliminer des obstacles isolés, ce qui a pour seuk résultat de modifier la section d'écoulement, mais il s'agira plutôt de coordonner les diverses mesures, et ainsi de chercher à compenser les pentes locales afin d'assurer en définitive un chenal navigable de la profondeur voulue. On effectuera également, si nécessaire, des essais sur modèle pour étudier tel ou tel endroit, et pour mettre le régime d'écoulement bien en évidence. Il sera vraisemblablement possible d'obtenir la profondeur navigable voulue de $2,10 \mathrm{~m}$ au-dessous de l'E.E. sur le parcours St. GoarMannheim, et de permettre ainsi aux bateaux de transporter jusqu'au Rhin-Supérieur, au Main et au Neckar la même charge qu'en aval de St. Goar.

Des travaux de régularisation complémentaires en aval de Coblence, à la tête amont du «Weissenthurmer Werth», seraient capables d'apporter des amélioration évitant les dragages 
qui sont encore assez souvent nécessaires. Cela mis à part, il suffirait d'entreprendre tout au plus quelques améliorations locales pour stabiliser certains ouvrages déjà construits.

Toutefois, sur le parcours en aval de Düsseldorf, il importera de toujours porter une attention toute particulière aux conséquences de l'érosion dont il a été parlé plus haut, et qui est toujours très active. On se propose de lutter contre cette érosion en continuant à abaisser les lits inondables et ceux des épis qui, implantés jadis à la cote des moyemnes eaux, se trouvent actuellement calés à une cote trop élevée.

Etant donné le volume important des travaux qu'elles nécessitent, ces mesures ne pourront se faire que par étapes, et ce surtout conjointement avec l'exploitation des graviers dans les lits inondables.
Plusieurs générations successives ont travaillé à la correction du Rhin, cette première voie fluviale d'Europe. Grâce à leurs efforts, les riverains de ce fleuve peuvent aujourd'hui vacuer à leurs diverses occupations et exploiter et cultiver les terres riveraines sans avoir à craindre sa puissance déchaînée.

Grâce à son caractère de grande artère de circulation, le Rhin est devenu un véritable « intermédiaire » entre les diver's pays d'Europe et pour leurs différentes économies, et il est encore loin d'avoir atteint la limite de ses possibilités en tant que voie de communication. C'est notre tâche que d'entretenir et prendre soin de ce qu'ont créé nos prédécesseurs et de l'adapter aux nécessités toujours plus diverses; cette même tâche sera également celle des générations futures.

Ros Recherchons :

\title{
INGENIEUR HYDRAULICIEN \\ en vue étude Projets Epuration
}

Adresser curriculum vilaë :

\author{
DEGREMONT-ACFI
}

Boîte Postale no 46 - suresnes (Seine) 